\title{
FAN BEAM AND PARALLEL BEAM PROJECTION
}

ANO BACK-PROJECTION OPERATORS

\author{
Grant T. Gullberq
}

Lawrence Berkeley Laboratory

University of Cal ifornia

Berkeley, California 94720

$$
\text { Januarv } 1977
$$

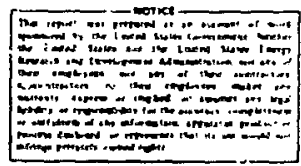

ABSTRACT

Expressions for the fan beam and parallel beam projection and backDrojection operators are given along with an cyaluation of the point source response for the back-projection operators. The tack-projection operator for the fan beam geometry requires the superposition of projection data measured over $360^{\circ}$. Both the fan beam and parallel beam geometries have back-projection operators with point source responses which are proportional to $1,1 \mathbf{r}-r_{0}$ ! and thus two-dimensional fourier filter techniques can be used to reconstruct transverse sections from fan beam and parallel beam projection data. The two-dimensional Fourier filter techniques may have the speed over other methods for reconstructing fan beam data but the reconstructed image requires four times the core storage so that the convolution result of one period does not overlap the convolution result of the succeeding period when implementing the fast Fourier transform. 
Abstract

1.0 INTRODUCTION

2.0 fan bEAM AND PARALlel bEAM PROJECTION OPERATORS ...... 4

2.1 The Equations for the Fan Beam and Parallel Beam

Projection Operators .............. 4

2.2 Projection of a Point Source . . . . . . . . . 9

2.3 Projection of a iircular Dijk . . . . . . . . . . 10

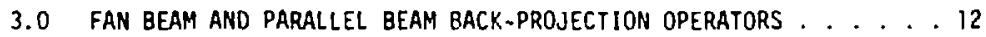

3.) Fan Beam Adjoint Projection Operator . . . . . . . . . 12

3.2 Paraliel Beam Adjoint Projection Operator . . . . . . 21

3.3 Other Back-Projection Operators . . . . . . . . . . 22

4.0 POINT SOURCE RESPONSE FOR BACK-PROJECTION OPERATORS .... . 24

4.1 The Back-Projection Operation Represented as a

Convolution with the Original Density ......... 24

4.2 Adjoint Transform of a Point Source Projection

Function for Fan Beam Geometry . . . . . . . . . . 25

4.2.1 The zeros a a

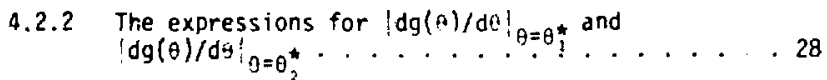

4.2.3 The expressions for $S\left(\theta_{i}^{*}\right)$ and $S\left(\theta_{?}^{*}\right) \ldots . . .29$

4.2.4 Solution to the transformed adjoint transform . . 32

4.3 Adjoint Transform of a Point Source Projection

Function for Parallel Beam Geometry ......... 39

4.4 The Back-Projection of a Fan Beam Point Source

Projection Function .............. 41

4.5 The Back-Projection of a Parallel Beam Point Source

Projection Function .....................

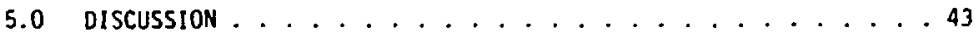

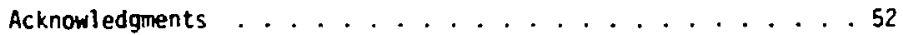

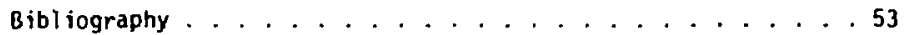




\subsection{INTRODUCTION}

Fan beam and parallel beam geometries have back-projection operators which accommodate the use of two-dimensional Fourier filter techniques for reconstructing transverse sections from projections. These back-projection operators give an image representing the true image convolved with a blurring function which for the parallel beam geometry equals $1 / r$. The relationship between the true and the back-projected inage is given by the equation

$$
b_{=}(r, 0)=f(r, \phi) * 1 / r
$$

where $b=$ is the back-projected image for parallel rays and $f$ is the true image. Budinger and Gullberg [1] conjectured that a similar result was also true for fan beam geometry and for beams of arbitrary orientations such as curved rays and randomly oriented chords from positron annihilation events. This report shows that this conjecture is true for fan beam geometry where the blurring function is equal $t, 2 / r$.

Using two-dimensional Fourier filter techniques the true image is reconstructed from the back-projected image for pavallel beam geometry by deconvolving the $1 / r$ blurring function using the relationship

$$
f(r, \phi)=\pi_{2}^{-1}|| R\left|T_{2}(b=\{r, \phi)\}\right| .
$$

This result was obtained by Bates, Peters and Smith $[2,3,4]$ and used in phantom studies by Budinger and Gullberg [1]. The results developed herein show that for the fan beam geometry the true image can be reconstructed from the back-projected image by deconvoiving the $2 / r$ blurring function 
using the relationship

$$
f(r, \phi)=\frac{1}{2} 7_{2}^{-1}\left\{|R| T_{2}\left\{b_{<}\{r, \phi)\right\}\right\}
$$

where $b_{<}(r, \phi)$ is the back-projected image for fan beam geometry.

The methods of reconstructing fan beam projection data can be divided into five categories:

1. Two-dimensional filtering of the back-projection.

This is developed in this report.

2. Re-ordering and rebinning the fan beam projection data so that the data correspond to a parallel beam grometry $[5,6]$. This allows the use of iterative methods, convolution methods, or Fourier space methods which are presently developed for reconstructing paralle? beam geometry.

3. Special convolution methode $[7,8,9]$. First a crsnvolution function is applied to the fan beam projection data and the result is then back-projected.

4. Radon's integration method $[10,11]$. The projection data are first differentiated, then followed by a Hilbert transform or an approximation using the trapezoidal method of integration.

5. Back-projection of the filtered fan beam projection data $[3,12]$. For each projection the data is first Fourier transformed, then filtered, nex: the inverse Fourier transform is taken and the result is tack-projected. Presently most of the methods being utilized for reconstructing fan beam data either rearrange the projection data so the datil correspond to parallel 
rays or use special convolution techniques which are adapted for fan beam geometry.

The text gives a detailed mathematical aralysis of fan beam and parallel beam projection and back-projection operators by first developing the expressions for the projection operators, next developing the expressions for the back-projection operators, and then evaluating the point source response for these back-projection operators. The results are sumarized in Figure 6, Section 5. 
2.0 FAN BEAM AND PARALLEL BEAM PROJECTION OPERATOKS

\subsection{The Equations for the Fan Beam and Parallel Beam Projection Operatiors}

The coordinate systems for the fan beam and parallel beam geometries are shown in figure 1 and Figure 2 respectively. The equations for the linear paths are dependent on their orientation relative to the axis of rotation. If we require that the vertex of the fan beam is left of the center of rotation as illustrated in Figure 1 , then the equation of the line emanating from the vertex in the $(x, y)$ coordinate system is

$$
R_{2} \sin \xi / R_{2}-x \sin \left(\omega-\xi / R_{2}\right)-y \cos \left(\theta-\xi / R_{2}\right)=0
$$

This expression can easily be derived if we first express the equation of the line in the $\left(x^{\circ}, y^{-}\right)$coordinate system,

$$
y^{*}=\tan \xi / R_{2} \times .
$$

With the coordinate transformation $(x, y) \rightarrow\left(x^{*}, y^{-}\right)$,

$$
\begin{aligned}
& x^{\prime}=x \cos \theta-y \sin \theta+R_{1} \\
& y^{\prime}=x \sin \theta+y \cos \theta .
\end{aligned}
$$

we can substitute equations (3) and (4) into equation (2) giving

$$
\begin{aligned}
& x \sin \theta+y \cos \theta=\tan \xi / R_{2}\left(x \cos \theta-y \sin \theta+R_{1}\right) \\
& x \sin \theta+y \cos \theta=\frac{\sin \xi / R_{2}}{\cos \xi / R_{2}}\left(x \cos \theta-y \sin \theta+R_{1}\right) \\
& x \sin \theta \cos \xi / R_{2}+y \cos \theta \cos \xi / R_{2}= \\
& \quad x \cos \theta \sin \xi / R_{2}-y \sin \theta \sin \xi / R_{2}+R_{2} \sin \xi / R_{2} .
\end{aligned}
$$

Collecting terms, we can express the above equation as

$$
\begin{array}{r}
R_{1} \sin \xi / R_{2}-x\left(\sin \theta \cos \xi / R_{2}-\cos \theta \sin \xi / \vec{R}_{2}\right) \\
-y\left(\cos \theta \cos \xi / R_{2}+\sin \theta \sin \xi / R_{1}\right)=0 .
\end{array}
$$


FAN BEAM GEOMETRY

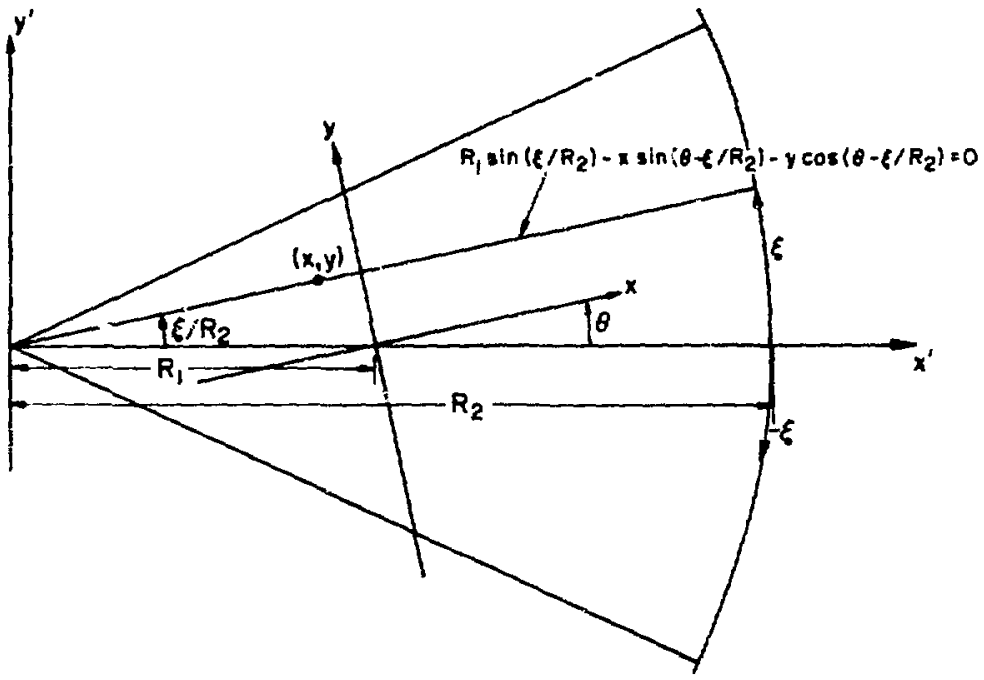

KBL767-9105:

Figure 1. The projection data $F_{<}(5, \theta)$ for a fan beam gcometry represent line integrals for the lines $R_{2} \sin \left(\xi / R_{2}\right)$. $x \sin \left(\theta-\xi / R_{2}\right)-y \cos \left(\theta-\xi / R_{2}\right)=0$. 
PARALLEL BEAM GEOMETRY

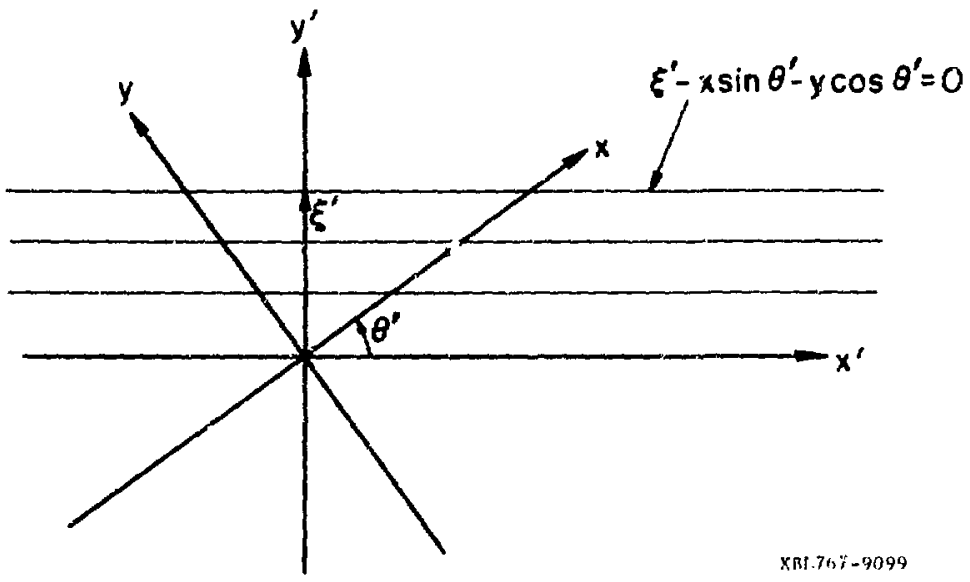

Figure 2. The projection data $p_{=}\left(F^{\circ}, n^{\circ}\right)$ for a parallel beam geometry represent line integrals for the ines $-x \sin i^{-}-y \cos 4^{-}=0$. 
Using the identities for the sine and cosine of the difference of two angles, equation (6) can be simplified giving the result in equation (1),

$$
\left.R_{2} \sin i / R_{:}-x \sin (i)-r / R_{2}\right)-j \cos \left(n-5, / R_{2}\right)=0 \text {. }
$$

The equations of the colinear lines for parallel beam geometry can be derived in a similar fashion. Another approach would be to keep $\left|R_{2}-R_{2}\right|$ fixed and allow $R_{1}$ and $R_{2}$ to approa: , i in equation (1) which in the limit gives the equation of a line for the parallel beam geometry,

$$
5^{\circ}-x \sin \theta^{\circ}-y \cos \theta^{\circ}=0 \text {. }
$$

The coordinate system for the parallel beam geometry is illustrated in Fig. 2 .

Using equation (1), the fan beam projection operator, $P_{<}$, is defined as $P,: f \cdot p$, where

$$
p_{<}\left(\xi_{,}, \theta\right)=\iint_{R^{2}} f(x, y) \delta\left(R_{1} \sin \xi_{\partial} / R_{2}-x \sin \left(\theta-\xi_{2} / R_{2}\right)-y \cos \left(\theta-\xi / R_{2}\right)\right) d x d y
$$

The integral is taken over $\mathbb{R}^{\prime}$ and it is assumed that $f(x, y)=0$ outside the cone spanned by the fan beam. The limits of the cone are a function of the length of the curved detector and a function of $R_{2}$ which is the distance between the vertex of the fan beam and the detector. Throughout this report we assume that the detector spans an arc between $-\pi R_{2} / 2$ and $\pi R_{2} / 2$. Using equation (7) the parallel beam projection operator, $P_{=}$, is defined as $P_{=}: f \rightarrow p_{=}$, where

$$
p_{=}\left(\xi^{\circ}, 0^{\circ}\right)=\iint_{\mathbb{R}^{2}} f(x, y) \delta\left(\xi^{-}-x \sin \theta^{-}-y \cos \theta^{\prime}\right) d x d y .
$$

The para?lel beam projection operator is linear and continuous when $f$ decreases rapidly at the boundary [13], and the projection function satisfies [14]

$$
p_{=}\left(\xi^{*}, \theta^{*}\right)=P_{=}\left(-\xi^{*}, \theta^{-}+\pi\right) \text {. }
$$


Likewise the fan beam projection operator is linear and continuous; however, the fan beam projection operator satisfies

$$
\mathrm{p}_{<}(\xi, \theta)=\mathrm{p}_{<}\left(-\xi, \theta+\pi-2 \xi ; \mathrm{R}_{2}\right) \text {. }
$$

The result given by equation (10) will become important when we consider back projection operators and the apprcpriate limits of integration.

The coordinates $\left(\xi^{-}, \theta^{*}\right)$ for the parallel beam projection function represent polar coordinates in the $x-y$ plane and are related to the coordinates $(x, y)$ through the transformation

$$
\begin{aligned}
& x=\xi^{\prime} \sin \theta^{\circ} \\
& y=\xi^{\prime} \cos \theta^{\circ} .
\end{aligned}
$$

However, the coordinates $(\xi, \theta)$ for the fan bean projection function do not represent polar coordinates in the $x-y$ plane but are related to the coordinates $(x, y)$ through the transformation

$$
\begin{aligned}
& x=R_{1} \sin \xi / R_{2} \sin \left(\theta-\xi / R_{2}\right) \\
& y=R_{1} \sin \xi / R_{2} \cos \left(\theta-\xi_{3} / R_{2}\right)
\end{aligned}
$$

and are related to the parallel beam projection coordinates $\left(\xi^{\circ}, \Theta^{-}\right)$through the transformation

$$
\begin{aligned}
& \xi^{-}=R_{1} \sin \xi / R_{2} \\
& \theta^{-}=\theta-\xi / R_{2}
\end{aligned}
$$

It is clear that the relationship between either the fan beam coordinates $(\xi, \theta)$ or the parallel beam coordinates $\left(\xi^{\prime}, \theta^{\prime}\right)$ and $(x, y)$ is not one-to-one since for any $\theta$ or $\theta^{-},(0, \theta)$ and $\left(0, \theta^{-}\right)$map onto $(x, y)=(0,0)$. The transformation given by equation (13) is user to reorganize the fan beam data into parallel beam data so that reconstruction algorithms applicable to parallel bean geometry can be lised $[5,6]$. 


\subsection{Projection of a Point Source}

The impulse response for the fan beam projection oderator can be evaluated for a point source at the position $\left(x_{0}, y_{0}\right)$ by substituting the two dìnensional delta function $f(x, y)=\delta\left(x-x_{0}\right) \delta\left(y-y_{0}\right)$ into equation $(8)$ giving

$$
\begin{aligned}
p_{<}(\xi, \theta) & =\iint_{\mathbb{R}^{2}} \delta\left(x-x_{0}\right) \delta\left(y-y_{0}\right) \delta\left(R_{2} \sin \xi / R_{2}-x \sin \left(\theta-\xi_{2} / R_{2}\right)-y \cos \left(\theta-\xi / R_{2}\right)\right) d x d y \\
& =\delta\left(R_{1} \sin \xi / R_{2}-x_{0} \sin \left(\theta-\xi / R_{2}\right)-y_{0} \cos \left(\theta-\xi / R_{2}\right)\right) .
\end{aligned}
$$

Substituting the expressions for $x_{0}$ and $y_{0}$ in terms of polar coordinates, $x_{0}=r_{0} \cos \phi_{0}$ and $y_{0}=r_{0} \sin \phi_{0}$, gives

$$
P_{<}(\xi, \theta)=\delta\left(R_{1} \sin \varepsilon / R_{2}-r_{0} \sin \left(\phi_{0}+\theta-\xi / R_{2}\right)\right)
$$

Similarly evaluating the projection of a point source for the parallel beam geometry gives

$$
P_{=}\left(\xi^{\prime}, \theta^{-}\right)=\delta\left(\xi^{-}-r_{0} \sin \left(\phi_{0}+\theta^{-}\right)\right) \text {. }
$$

Later we will see that we can rewrite equation (14) as

$$
p_{<}(\xi, \theta)=\frac{R_{2} \delta\left(\xi-R \tan ^{-3}\left[\frac{r_{0} \sin \left(\phi_{0}+\theta\right)}{R_{1}+r_{0} \cos \left(\phi_{0}+\theta\right)}\right]\right)}{\sqrt{R_{1}^{2}+r_{0}^{2}+2 r_{0} R_{1} \cos \left(\phi_{0}+\theta\right)}}
$$

If we integrate $p_{<}(\xi, \theta)$ over $\xi$ we find that

$$
\begin{aligned}
\int_{\mathbb{R}} P_{<}\{\xi, \theta) d \xi & =\frac{R_{2}}{\sqrt{R_{1}^{2}+r_{0}^{2}+2 r_{0} R_{1} \cos \left(\phi_{0}+\theta\right)}} \\
& =\frac{\text { Distance from fan source to detector }}{\text { Distance from fan source to }\left(x_{0}, y_{0}\right)}
\end{aligned}
$$




$$
\int_{\mathbb{R}} P_{=}\left(\xi^{\prime}, \theta^{-}\right) d \xi^{\prime}=1 .
$$

\subsection{Projection of a Circular Disk}

For the fan beam projection operator we consider the projection of a circular disk, $f(x, y)$, defined as

$$
f(x, y)= \begin{cases}1 & x^{2}+y^{2} \leqslant R<R_{1} \\ 0 & \text { otherwise }\end{cases}
$$

Since the projection of a circular disk is independent of angle, the projection of $f(x, y)$ is just the length of a cord going through the circle as illustrated in Figure 3.

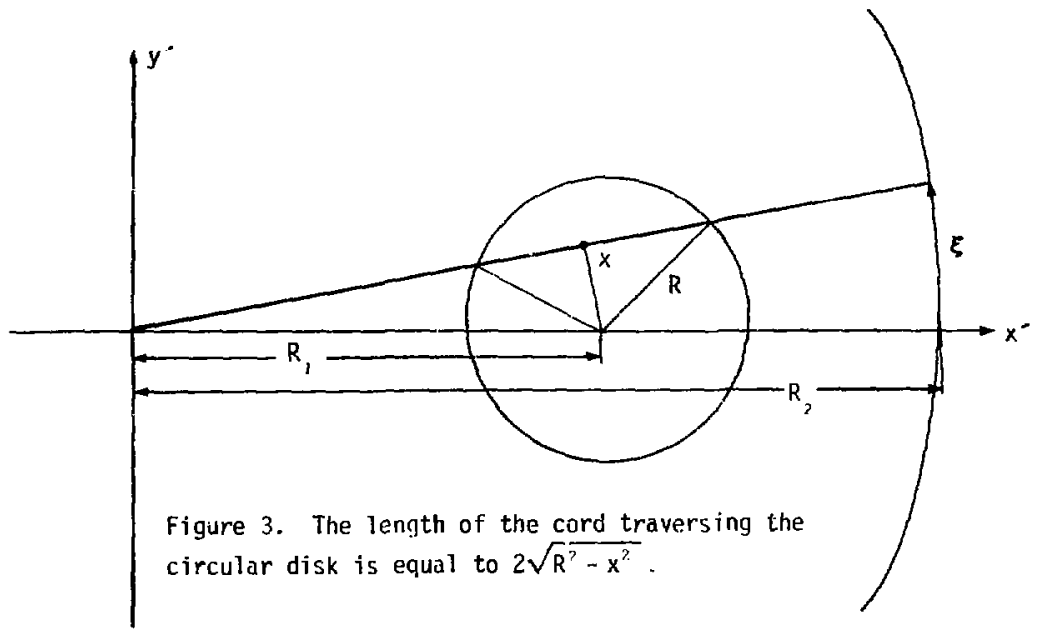

The length of the cord subtending the circle is

$$
2 \sqrt{R^{2}-x^{2}}
$$

where $x$ is the perpendicular distance from the center of rotation to the 
line. Substituting the expression of $x$ in terms of $\xi$,

$$
x=R_{1} \sin \xi / R_{2}
$$

into equation (16) gives the projection of the circular disk, $f(x, y)$, for the fan beam geometry,

$$
P_{<}(\xi, \theta)= \begin{cases}2 \sqrt{R^{2}-R_{1}^{2} \sin \xi / R_{2}} & \left|\sin \xi / R_{2}\right| \leqslant R / R_{1} \\ 0 & \text { otherwise. }\end{cases}
$$

Using the transformation given by equation (13) gives

$$
P_{=}\left(\xi^{\prime}, \theta^{\prime}\right)=\left\{\begin{array}{ll}
2 \sqrt{R^{2}-\xi^{-2}} & \left|\xi^{\prime}\right| \leqslant R \\
0 & \text { otherwise. }
\end{array} .\right.
$$

The expression given in equation (17) is equal to the parallel beam projection function where $\xi_{2}^{\prime}$ is identical to $x$ and represents the closest distance between the line and the center of rotation. 


\subsection{FAN BEAM AND PARALLEL BEAM BACK-PROJECTION OPERATORS}

\subsection{Fan Beam Adjoint Projection Operator}

The adjoint operator $P_{<}^{+}$of the fan beam projection operator $P_{c}$ is by definition the operator which satisfies the relationship

$$
\left\langle P_{<} f, P_{<}\right\rangle=\left(f, P_{<}^{+} P_{<}\right)
$$

where $($,$) denotes an inner product defined as$

$$
(f, g) \quad \iint_{\mathbb{R}^{2}} f(x, y) g(x, y) d x d y .
$$

We will show in this section that the adjoint operatur $P_{c}^{+}: p_{e} \rightarrow p_{c}^{+}$is defined by the equation

$$
p_{<}^{+}(r, \phi)=\int_{-\phi}^{\pi-\phi} \frac{R_{1}^{2} p_{<}\left(\xi^{*}, \theta\right) r \sin (\theta+\phi)\left[R_{1}+r \cos (\phi+\theta)\right]}{\left[r^{2}+R_{1}^{2}+2 R_{1} r \cos (\phi+\theta)\right]^{3 / 2}} d \theta
$$

where

$$
\xi^{*}=R_{2} \tan ^{-1}\left[\frac{r \sin (\theta+\phi)}{R_{1}+r \cos (\theta+\phi)}\right]
$$

The kernel of the adjoint transformation satisfies the equation

$$
K\left(R_{1}, r, \phi, \theta\right)=\frac{R_{1}^{2} r \sin (\theta+\phi)\left[R_{1}+r \cos (\phi+\theta)\right]}{\left[r^{2}+R_{1}^{2}+2 R_{1} r \cos (\phi+\theta)\right]^{3 / 2}}
$$

If we eliminate the kernel $K\left(R_{1}, r, \phi, \theta\right)$ from the expression for the adjoint operator and extend the limits of integration for 0 to be from 0 to $2 \pi$, then we obtain the fan beam back-projection operator, $B_{<}: p_{<} \rightarrow b_{<}$, defined by the expression 
$\left.D,\{r, \theta)=\int_{0}^{2 \pi} p_{r}(r, 0) d r\right)$

The adjoint operator of the projection operator and the back-projection operator are members of a class of "back-projection" operators which give the superposition of the projection line integrals weighted by a particular kernel which for the back-projection operator is equal to the identity function.

In Section 3.2 we will develop the adjoint operator for the parallel beam projection operator [13] which satisfies the relationship

$$
\left.p_{0}^{+}(r, \phi)=\int_{0}^{\pi} p_{=}\left(r \sin (\phi+\pi), 0^{\circ}\right) \operatorname{rsin}\left(\theta+\theta^{\circ}\right)\right) d \theta^{\circ} .
$$

Removing the kernel irsin( $+\cdots)$ from the integrand gives the expression for the parallel beam bdck-projection operator, $B_{=}: P_{=} \cdot b_{=}$, defined by the equation

$$
b_{=}(r, \theta)=\int_{0}^{\pi} p_{=}\left(r \sin (\theta+0), 0^{\circ}\right) d \theta^{\circ} .
$$

The back-projection operator for parallel beam geometry has been defined by various authors - Peters [3] identified the back-projection operator with the layergram, Gllbert [15] called it back-projection, and Budinger and Gillberg [16] identified it with linear superposition.

For the digital implementation of the projection and back-projection operation we use a projection operator represented by the matrix $\oint_{=}$which maps the transverse section vector $\mathbb{A}$ into the projection vector $\mathbb{P}$ according to the relationship

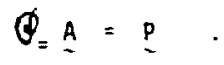


The adjoint operator of this projection operator is equal to the transpose of the projection operator.

$$
Q_{=}^{+}=Q_{z}^{\mathrm{T}}
$$

The back-projection operator used for the digital application of the backprojection operation is equal to the adjoint operator as indicated by the expression

$$
B==Q_{=}^{+}=Q_{=}^{T}
$$

This is in contrast to the continuous situation where the back-projection and adjoint operator are not identical. The back-projection approach to estimating the original density from its projection, as given by the equation

$$
\underline{B}=B_{=} \stackrel{p}{\sim}=Q_{=}^{T} \stackrel{p}{\sim}
$$

where $B$ is the back-projection vector, is the most rapid method of reconstruction but gives an image which is equal to the original density convolved with $1 / r$.

To develop the expression for the adjoint operator of the fan beam projection operator we first evaluate the inner product in equation (18)

$$
\left(p_{<} f, p_{<}\right)=\iint_{\mathbb{R}^{2}} P_{<} f\left(x^{*}, y^{\prime}\right) p_{<}\left(x^{*}, y^{\prime}\right) d x^{*} d y^{*}
$$

Substituting the expression for the projection operator of equation (8) into equation (20) gives the relationship

$$
\begin{aligned}
\left\langle P_{<} f, p_{<}\right\rangle= & \int_{0}^{2 \pi} \int_{0}^{R_{2} \pi / 2} \int_{\mathbb{R}^{2}} f(x, y) p_{<}(\xi, \theta) x \\
& \delta\left(R_{1} \sin \xi / R_{2}-x \sin \left(\theta-\xi / R_{2}\right)-y \cos \left(\theta-\xi / R_{2}\right)\right) d x d y|d| d \xi d \theta
\end{aligned}
$$


where $d x^{\circ} d y^{*}$ is replaced by $|J| d \xi d \theta$ and $J$ is the Jacobian for the transformation relating the coordinates $\left(x^{*}, y^{*}\right)$ to the coordinates $(\xi, \theta)$ given by equation (12),

$$
\begin{aligned}
& x^{\circ}=R_{1} \sin \gamma / R_{2} \sin \left(r-5 / R_{2}\right) \\
& y^{-}=R_{1} \sin r / R_{2} \cos \left(\theta-5 / R_{2}\right) .
\end{aligned}
$$

In equation (21) the limits of integration for $\xi$ and for $\theta$ were chosen so that the space $\mathbb{R}^{2}$ is properly sampled. Figure 4 gives the trace of the locus of noints representing the projected coordinates of a point source as it is ratated from 0 to 360 degrees. The trace for the parallel beam geometry illustrates that $p_{n}\left(5^{*}, 0^{\circ}\right)=p_{0}\left(-5^{\circ}, \pi+0^{\circ}\right)$; therefore, we can always properly sample the projection space if we integrate $\xi$ between $-\infty$ and $+\infty$, and $n$ between 0 and $\pi$. However, for the fan beam geametry the trace illustrates that $p_{<}\left(\xi_{,}, 0\right)=p_{<}\left(-E_{3}, C+\pi-2 c_{2} / R_{2}\right)$ and in order to properly sample the space $\mathbb{R}^{i}$ we need to choose the limiis of integration for $F$, between 0 and $R_{2} \pi / 2$ and for $\theta$ between 0 and $2 \pi$. In choosing the 1 imits for $\xi$, we assume that the detector for the fan beam spans an arc between $-R_{2} \pi / 2$ and $R_{2} \pi / 2$.

The Jacobian for the transformation given above is the determinant

$$
J=\frac{\partial\left(x^{*}, y^{*}\right)}{\partial(\xi, 0)}=\left|\begin{array}{ll}
\frac{\partial x^{*}}{\partial \xi} & \frac{\partial x^{\circ}}{\partial \theta} \\
\frac{\partial y^{\prime}}{\partial \xi} & \frac{\partial y^{*}}{\partial \theta}
\end{array}\right|
$$

where

$$
\begin{aligned}
& \frac{\partial x^{\prime}}{\partial \xi}=\frac{R_{1}}{R_{2}} \sin \left(\theta-2 \xi / R_{2}\right), \\
& \frac{\partial x^{\circ}}{\partial \theta}=R_{1} \sin \xi / R_{2} \cos \left(\theta-\xi / R_{2}\right),
\end{aligned}
$$


SINOGRAM OF Q POIMT SOURCE FOR

FAN BEAM AND PAEALLEL BEAM GEUMETRY

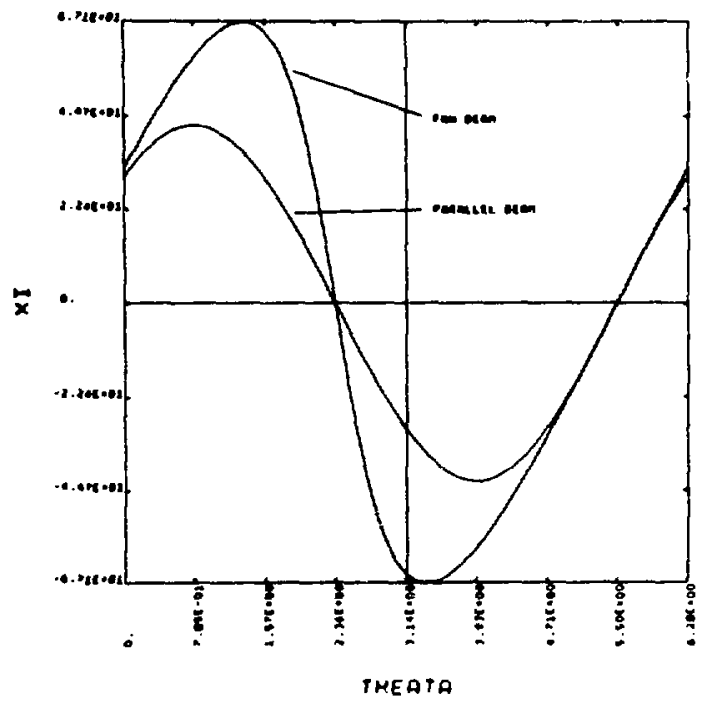

POINT SOURCE CODROINATES - 130.301

FAN BEAM UERTEX COMROINRTES - R1 $=B O, R 2=120$

Figure 4. The locus of points representing the coordinates $(\xi, \theta)$ and $\left(\xi^{\prime}, \theta^{2}\right)$ for a projected point source for fan beam and parallel beam geometry, respectively. 


$$
\begin{aligned}
& \frac{\partial y^{\circ}}{\partial F_{0}}=\frac{R_{1}}{R_{2}} \cos \left(\theta-2 \xi_{3} / R_{2}\right) . \\
& \frac{\partial y^{\circ}}{\partial \theta}=-R_{1} \sin \xi / R_{2} \sin \left(\theta-\xi / R_{2}\right) .
\end{aligned}
$$

Evaluating the determinant in equation (22) gives

$$
J=\frac{\partial x^{*}}{\partial \xi_{3}} \frac{\partial y^{-}}{\partial \bar{\theta}^{-}} \cdots \frac{\partial x^{*}}{\partial \theta} \frac{\partial y^{*}}{\partial \xi}=\frac{-R_{1}^{2}}{R_{2}} \sin \xi / R_{2} \cos \xi / R_{2} .
$$

Returning to equation (21), we can rewrite the inner product by interchanging the integrals

$$
\begin{aligned}
\left\langle P_{<} f, P_{<}\right\rangle & =\iint_{\mathbb{R}^{2}} f(x, y) \int_{0}^{2 \pi} \int_{0}^{R_{2} \pi / 2} p(\xi, \theta) \delta\left(R_{1} \sin \xi / R_{2}-x \sin \left(\theta-\xi / R_{2}\right)\right. \\
& \left.-y \cos \left(\theta-\xi / R_{2}\right)\right) ! J \mid d \xi d \theta d x d y,
\end{aligned}
$$

provided the functions $f$ and $p_{<}$satisfy the conditions of Fubini's theorem $[17, p .323]$, i.e. $f$ and $p_{c}$ are Lebesque integrable on a closed subset of $\mathbb{R}^{2}$. This is always satisfied in real problems since $f$ and $p_{<}$are continuous functions almost everywhere and bounded functions on a closed subset of $\mathbb{R}^{?}$. In order to agree with equation (18), we take the inner double integral in equation (24) and define the adjoint operator of the fan beam projection operator as

$$
\begin{aligned}
P_{<}^{+} p_{<} & =p_{i}^{+}(x, y)=\int_{0}^{2 \pi} \int_{0}^{R_{2} \pi / 2} p_{<}\left(E_{2}, \theta\right) \delta\left(R_{1} \sin \xi / R_{2}-x \sin \left(\theta-\xi / R_{2}\right)\right. \\
& \left.-y \cos \left(0-E_{1} / R_{2}\right)\right)|J| d \xi_{2} d \theta
\end{aligned}
$$

Equation (25) can be simplified by int-grating over the variable $\xi$. In order to do this we use the relationship $[18, p .38]$ 


$$
\int_{0}^{\pi R_{2} / 2} p_{<}(\xi, \theta) \delta(h(\xi))|\mathrm{J}(\xi)| d \xi=\int_{0}^{\pi R_{2} / 2} \sum_{i} p_{<}(\xi, \theta) \frac{\delta\left(\xi-\xi_{j}^{*}\right)}{\left|\frac{\mathrm{dh}(\xi)}{\mathrm{d} \xi_{i}}\right|_{\xi=\xi_{i}^{*}}}|J(\xi)| d \xi
$$

where $J(\xi)$ indicates that the Jacobian is a function of $\xi$ and $\xi_{i}^{*}$ are the zeros of $h$,

$$
h(\xi)=R_{1} \sin \xi / R_{2}-x \sin \left(\theta-\varepsilon / R_{2}\right)-y \cos \left(\theta-\xi / R_{2}\right) .
$$

The function $h$ has a unique zero $\xi^{\star}$ which satisfies

$$
\begin{aligned}
\xi^{*} & =R_{2} \tan ^{-1}\left[\frac{x \sin \theta+y \cos \theta}{R_{1}+x \cos \theta-y \sin \theta}\right], \\
& =R_{2} \tan ^{-1}\left[\frac{r \sin (\theta+\phi)}{R_{1}+r \cos (\theta+\phi)}\right]
\end{aligned}
$$

and $\sin \xi^{\star} / R_{2}$ and $\cos \xi^{\star} / R_{2}$ satisfy

$$
\begin{aligned}
& \sin \xi^{*} / R_{2}=\frac{x \sin \theta+y \cos \theta}{\left[R_{1}^{2}+x^{2}+y^{2}+2 R_{1}(x \cos \theta-y \sin \theta)\right]^{\frac{1}{2}}}=\frac{r \sin (\phi+\theta)}{\left[r^{2}+R_{1}^{2}+2 R_{1} r \cos (\phi+\theta)\right]^{\frac{1}{2}}} \\
& \cos \xi^{*} / R_{2}=\frac{R_{1}+x \cos \theta-y \sin \theta}{\left[R_{1}^{2}+x^{2}+y^{2}+2 R_{1}(x \cos \theta-y \sin \theta)\right]^{\frac{1}{2}}}=\frac{R_{1}+r \cos (\phi+\theta)}{\left[r^{2}+R_{1}^{2}+2 R_{1} r \cos (\phi+\theta)\right]^{\frac{1}{2}}}
\end{aligned}
$$

where the polar coordinates $x=r \cos \phi, y=r \sin \phi$ are substituted for $x$ and $y$. Integrating the expression in equation (26) gives 


$$
\int_{0}^{\pi ?{ }_{2} / 2}
$$

$F,(\xi, \theta) \delta(h(\xi)) \backslash J(\xi)) d \xi=$

$$
\begin{cases}\frac{p_{\zeta}\left(\xi^{*}, 0\right) J\left(\xi^{*}\right)}{\left.\frac{d h(\xi)}{d \xi}\right|_{\xi=\xi^{*}}} & \text { if } \xi^{*}>0 \\ 0 & \text { otherwise }\end{cases}
$$

From equation (28) we see that if $R_{1}$ is greater than $r$, then the condition $\xi^{*}>0$ can be replaced by $|0+\phi|<\pi$.

In order to evaluate the denominator in equation (31), we take the derivative of $h$ giving

$$
\left|\frac{d h(\xi)}{d \xi}\right|_{\xi=?^{*}}=\left|\frac{R_{1}}{R_{2}} \cos \xi / R_{2}+\frac{x}{R_{2}} \cos \left(\theta-\xi / R_{2}\right)-\frac{y}{R_{2}} \sin \left(\theta-\xi / R_{2}\right)\right|_{\xi=\xi^{*}} .
$$

Expanding and collecting the tems which are factors of $\sin \xi / R_{2}$ and $\cos \xi / R_{2}$ gives

$$
\frac{d h(\xi)}{d \xi}=\frac{\left(R_{1}+x \cos \theta-y \sin \theta\right)}{R_{2}} \cos E_{1} / R_{2}+\frac{(x \sin \theta+y \cos \theta)}{R_{2}} \sin \xi / R_{2}
$$

and in polar coordinates gives

$$
\frac{d h(\xi)}{d \xi}=\left[\frac{R_{1}+r \cos (\phi+\theta)}{R_{2}}\right] \cos \xi / R_{2}+\frac{r}{R_{2}} \sin (4+\theta) \sin \xi / R_{2} .
$$

Substituting the value of $\sin \xi^{\star} / R_{2}$ and $\cos \xi^{\star} / R_{2}$ given by equations (29) and (30) gives

$$
\begin{aligned}
\left|\frac{d h(\zeta)}{d \xi}\right|_{\xi=\xi^{*}} & =\left|\frac{\left[R_{1}+r \cos (\phi+\theta)\right]^{2}}{R_{2}\left[r^{2}+R_{1}^{2}+2 R_{1} r \cos (\phi+\theta)\right]^{\frac{1}{2}}}+\frac{r^{2} \sin ^{2}(\phi+\theta)}{R_{1}\left[r^{2}+R_{1}^{2}+2 R_{1} r \cos (\phi+\theta)\right]^{\frac{1}{2}}}\right| \\
& =\left[r^{2}+R_{1}^{2}+2 R_{1} r \cos (\phi+\theta)\right]^{\frac{1}{2}} / R_{2} .
\end{aligned}
$$


Substituting equation (32) into equation (31) and replacing the condition $E_{1}^{*}>0$ with $-\phi<\theta<\pi-\phi$ gives the expression

$$
\int_{0}^{\pi R_{2} / 2} \rho_{<}(\xi, \theta) \delta(n(\xi))|J(\xi)| d \xi= \begin{cases}\frac{R_{2} \mu_{<}\left(\xi^{\star}, \theta\right) \mid J\left(\xi^{\star}\right) !}{\left[r^{2}+R_{1}^{2}+2 R_{1} r \cos (\phi+\theta)\right]^{\frac{1}{2}}} & \text { if }-\phi<\theta<\pi \phi \\ 0 & \text { otherwise }\end{cases}
$$

We can now give an expression for the adjoint operator $p_{<}^{+}$by substituting equation (33) and the expression for the Jacobian [equation (23)] into equation (25) giving

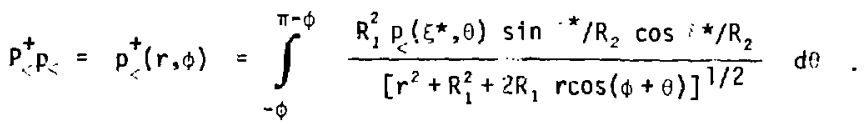

The absolute value sign is removed since both $\sin \xi^{\star} / R_{2}$ and $\cos \xi^{\star} / R_{2}>0$ for $-\phi<\theta<\pi-\phi$ and $R_{1}>r$. If we substitute for $\sin \xi^{\star} / R_{2}$ and $\cos \xi^{\star} / R_{2}$ given in equations (29) and (30), the adjoint operator is

$$
P_{-}^{+} p_{c}=p_{c}^{+}(r, \phi)=\int_{-\phi}^{\pi-\phi} \frac{R_{1}^{2} p_{c}\left(\xi \xi^{\star}, \theta\right) r \sin (\theta+\phi)\left[R_{1}+r \cos (\phi+\theta)\right]}{\left[r^{2}+R_{1}^{2}+2 R_{2} r \cos (\phi+\theta)\right]^{3 / 2}} d \theta
$$

where $K\left(R_{1}, r, \phi, \theta\right)$ is given by equation (19) and the expression for $\xi^{*}$ is given by equation (28).

The adjoint operator given by equation (34) represents the integration over all projection values $p_{<}\left(\xi^{\star}, \theta\right)$ which pass through the point $(r, \phi)$ weighted by the kernel $k\left(R_{1}, r, \phi, \theta\right)$. Thererure this adjoint operator is a 
memter of the class of "back-proiection" operators. It will be proved later that if the kernel is removed, we can define a back-projection operator which gives a response proportional to $1 / r$ for a point source projection function.

\subsection{Parallel Beam Adjoint Projection Operator}

The adjoint projection operator for the parallel beam geometry can be derived using the same methods developed in evaluating the fan beam adjoint projection operator. From Fig. 2 we see that the coordinate transformation relating $(x, y)$ to $\left(\xi^{\circ}, 0^{\circ}\right)$ is

$$
\begin{aligned}
& x=\xi^{\prime} \cos \left(\pi / 2-\theta^{\prime}\right) \\
& y=\xi^{\circ} \sin \left(\pi / 2-\theta^{\circ}\right)
\end{aligned}
$$

and the Jacobian for this transformation is $J=-\xi^{\circ}$. Therefore the adjoint projection operator defined in equation (25) for fan beam geometry can also be defined for parallel beam geotietry using the equation

$$
p_{=}^{+} p_{=}=p_{=}^{+}(x, y)=\int_{0}^{\pi} \int_{-\infty}^{\infty} p_{=}\left(\xi^{\circ}, \theta^{-}\right) \delta\left(\xi^{\circ}-x \sin \theta^{-}-y \cos \theta^{-}\right)\left|-\xi^{-}\right| d \xi^{\prime} d \theta^{\circ} \text {, }
$$

or in polar coordinates using the equation

$$
P_{=}^{+} p_{=}=p_{=}^{+}(r, \phi)=\int_{0}^{\pi} \int_{-\infty}^{\infty} p_{=}\left(\xi^{\prime}, \theta^{-}\right) \delta\left(\xi^{-}-r \sin \left(\phi+\theta^{-}\right)\right)\left|\xi^{-}\right| d \xi^{-} d \theta^{-} \text {. }
$$

If we integrate over $\xi^{\circ}$ in equation (35) then the adjoint projection operator for parallel beam geometry is

$$
P_{=}^{+} p_{=}=p_{=}^{+}(r, \phi)=\int_{0}^{\pi} p_{=}\left(r \sin \left(\phi+\theta^{-}\right), \theta^{\prime}\right)\left|r \sin \left(\phi+\theta^{-}\right)\right| d \theta^{\circ} .
$$




\subsection{Other Back-Projection Operators}

Ein-Gal [13] defines two other transforms for parallel beam geometry which are "back-projection" operators similar to the adjoint transform. One of these operators is the unweighted integration of $P_{=}\left(\xi^{\circ}, \theta^{\prime}\right)$ along the circle $C$ shawn in Fig. 5 ,

$$
c(r, \phi)=\int_{0}^{\pi} p_{=}\left(r \sin \left(\theta^{\circ}+\phi\right), \theta^{\circ}\right) r d \theta^{\circ} .
$$

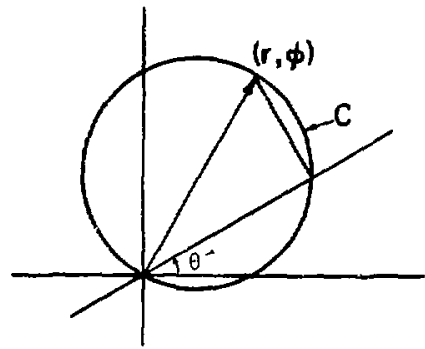

Figure 5. The value for the back-projection at the point $(r, \phi)$ represents integration along the circle $c$.

Ein-Gal also defines another back-jrojection operator which is the transformation of the scaled projection function,

$$
s(r, \phi)=\int_{0}^{\pi} p_{=}\left(r \sin \left(\phi+\theta^{\circ}\right), \theta^{\prime}\right) \frac{d \theta^{\circ}}{\left|\sin \left(\phi+\theta^{-}\right)\right|} .
$$

Here again the integration is performed along the circle $C$ in Fig. 5 but this time the projection values are scaled by the factor $1 /\left|\sin \left(\phi+\theta^{\prime}\right)\right|$.

Another back-projection operator for parallel beam geometry is

$$
B_{z} p_{=}=b_{=}(r, \phi)=\int_{0}^{\pi} p_{=}\left(r \sin \left(\phi+\theta^{-}\right), \theta^{\prime}\right) d \theta^{\circ} .
$$


Peters [3] has identified this as the layergram, others have defined this as Tinear superposition [16] or just back-projection [15]. Notice that in this definition the differential arc length, $r d \theta^{\prime}$, for the circle $C$ in equation (37) is replaced by $d \Theta^{\circ}$. The result of this transform applied to a point source projection function gives

$$
B=\delta\left(\xi^{\prime}-r_{0} \sin \left(\phi_{0}+\theta^{-}\right)\right)=b_{=}(r, \phi)=\frac{1}{\left|r-r_{0}\right|} .
$$

For fan beam geometry, if we define a back-projection operator

$$
B_{<} p_{<}=b_{<}(r, \phi)=\int_{0}^{2 \pi} p_{<}\left(\xi^{\star}, \theta\right) d \theta
$$

where $\xi^{\star}$ is given by equation $(28)$, then $b_{<}(r, \phi)$ has a similar response for a point source projection function as does the parallel beam geometry [equation (40)],

$$
B_{<} \delta\left(R_{1} \sin \xi / R_{2}-r_{0} \sin \left(\phi_{0}+\theta-\xi / R_{2}\right)\right)=b_{<}\langle r, \phi\}=\frac{2}{\left|r-r_{0}\right|} .
$$

The kernel $n^{\prime}\left(R_{1}, r, \phi, \theta\right)$ in the definition of the fan beam adjoint projection operator given in equa ${ }^{\wedge}$ ion (34) does not appear in the integrand for the definition of the fan beam back-projection operator [equation (41)]. The proof of the result in equation (42) will be given in the next section. The results in equations (40) and (42) are desirable since it means there exists back-projection operators both for the fan beam and parallel beam geometries which represent the original density convolved with a function proportional to $1 / r$. Therefore, two-dimensional Fourier filter techniques can be applied for both fan beam and parallel beam geometries to obtain a reconstructed image from its projections. 


\subsection{POINT SOURCE RESPONSE FOR BACK-PROJECTION OPERATORS}

\subsection{The Back-Projection Operation Represented as a Convolution}

\section{with the Original Density}

In order to prove that a back-projection operation represents a convolution with the original density $f(x, y)$ we need only to evaluate the back-projection of a point source projection function. To illustrate why this is so, let us consider writing the original density $f(x, y)$ as

$$
f(x, y)=\iint f\left(x^{\prime}, y^{\prime}\right) \delta\left(x-x^{\prime}\right) \delta\left(y-y^{\prime}\right) d x^{\prime} d y^{\prime} .
$$

Then applying a projection operator, $P: f \rightarrow p$, we have

$$
p(\xi, \theta)=\iiint \int f\left(x^{\prime}, y^{\prime}\right) \delta\left(x-x^{\prime}\right) \delta\left(y-y^{\prime}\right) d x^{-} d y^{-} \delta(g(x, y, \xi, \theta)) d x d y
$$

where $g(x, y, \xi, \theta)$ represent some curvilinear path. Then assuming that the conditions are met which allow us to interchange the integrals, we can integrate over $x$ and $y$, giving

$$
p(\xi, \theta)=\iint f\left(x^{\prime}, y^{\prime}\right) \delta\left(g\left(x^{\prime}, y^{\prime}, \xi, \theta\right)\right) d x^{-} d y^{-} .
$$

The delta function $\delta\left(g\left(x^{*}, y^{*}, \xi, \theta\right)\right)$ represents the projection of a point source. Then applying a back-projection operator, $B: p+b$, gives

$$
b(x, y)=\iiint f\left(x^{*}, y^{-}\right) \delta\left(g\left(x^{-}, y^{*}, \xi^{\star}, \theta\right)\right) d x^{*} d y^{*} d \theta
$$

where $\xi^{\star}$ is a function of $x, y$ and $\theta$. Interchanging the order of integration gives

$$
b(x, y)=\iint f\left(x^{-}, y^{-}\right)\left[\int \delta\left(g\left(x^{-}, y^{-}, \xi^{\star}(x, y, \theta), \theta\right)\right) d \theta\right] d x^{-} d y^{-}
$$


where

$$
\int \delta\left(g\left(x^{*}, y^{*}, \xi^{\star}(x, y, \theta), \theta\right)\right) d \theta
$$

represents the point source response to the projection - back-projection operation. Therefore if $b(x, y)$ is to be represented as a convolution, the point source response must be a function that can be represented as $h\left(x-x^{*}, y-y^{-}\right)$. We will first show that such a functional representation does not exist for the adjoint transform of a projected point source both for the fan beam and parallel beam geometry. Then we will show that the back-projection operators given by equations (39) and (41) represent a convolution of the original density with a function proportional to $1 / \mathrm{r}$.

\subsection{Adjoint Transform of a Point Source Projection Function for}

\section{Fan Beam Geometry}

The adjoint transform of a point source projection function is evaluated by substituting the fan beam projection function for a point source given in equation (14) into equation (34) giving

$$
P_{<}^{+} \delta=P_{\tau}^{+}(r, \phi)=\int_{-\phi}^{\pi-\phi} \frac{R_{1}^{2} \delta\left(R_{1} \sin \xi^{\star} / R_{2}-r_{0} \sin \left(\phi_{0}+\theta-\xi^{\star} / R_{2}\right)\right) r \sin (\phi+\theta)\left[R_{1}+r \cos (\phi+\theta)\right]}{\left[r^{2}+R_{1}^{2}+2 R_{1} r \cos (\phi+\theta)\right]^{3 / 2}} d \theta \text {. }
$$

If we let $g(\theta)$ denote the argument in the del ta finction in equation (43), then we can substitute for $\xi^{\star}$ using equations (29) and (30),

$$
\begin{aligned}
g(\theta) & =\left(R_{1}+r_{0} \cos \left(\phi_{0}+\theta\right)\right) \sin \xi^{\star} / R_{2}-r_{0} \sin \left(\phi_{0}+\theta\right) \cos \xi^{\star} / R_{2} \\
& =\frac{R_{1}\left(r \cos \phi-r_{0} \cos \phi_{0}\right) \sin \theta+R_{1}\left(r \sin \phi-r_{0} \sin \phi_{0}\right) \cos \theta+r_{0} r \sin \left(\phi-\phi_{0}\right)}{\left[r^{2}+R_{1}^{2}+2 R_{1} r \cos (\phi+\theta)\right]^{1 / 2}} .
\end{aligned}
$$


Rewriting $g(\theta)$,

$$
g(\theta)=\frac{a \cos \theta+b \sin \theta+c}{s^{1 / 2}}
$$

where

$$
\begin{aligned}
& a=R_{1}\left(r \sin \phi-r_{0} \sin \phi_{0}\right)=R_{1}\left(y-y_{0}\right) \\
& b=R_{1}\left(r \cos \phi-r_{0} \cos \phi_{0}\right)=R_{1}\left(x-x_{0}\right) \\
& c=r_{0} r \sin \left(\phi-\phi_{0}\right)=x_{0} y-x y_{0} \\
& S=r^{2}+R_{1}^{2}+2 R_{1} r \cos (\phi+\theta)
\end{aligned}
$$

and substituting into equation (43) gives

$$
p_{<}^{+}(r, \phi)=\int_{-\phi}^{\pi-\phi} \frac{R_{1}^{2} \delta(g(\theta)) r \sin (\theta+\phi)\left[R_{1}+r \cos (\phi+\theta)\right]}{s^{3 / 2}} d \theta .
$$

To evaluate equation (46) we need to determine the zeros $0_{i}^{*}$ of $g(\theta)$ as we did in equation (28) for $h(i)$ and evaluate $|d g(\theta) / d \theta|_{\theta=3 k_{j}^{*}}$ This then allows us to rewrite equation (46)

$$
p_{<}^{+}(r, \phi)=\int_{-\phi}^{\pi-\phi} \frac{R_{1}^{2} r \sin (\theta+\phi)\left[R_{1}+r \cos (\phi+\theta)\right]}{s^{3 / 2}}\left(\sum_{i} \frac{\delta\left(\theta-\theta_{i}^{\star}\right)}{\left|\frac{d g(\theta)}{d \theta}\right|_{\theta=\theta_{i}^{*}}}\right) d \theta .
$$

\subsubsection{The zeros $\theta_{1}^{*}$ and $\theta_{2}^{*}$ of $g(\theta)$}

Let us determine the zeros of $g(1)$ by first setting $u=$ sin" and writing $g(i)$ as

$$
g(u)=\frac{ \pm a \sqrt{1-u^{2}}+b u+c}{s^{1 / 2}} .
$$


Determining the zerus of $g(u)$ is equivalent to evaluating the solution to the equation

$$
\text { - } a \sqrt{1-u^{2}}+b u+c=0 \text {. }
$$

The solution of equation (47) is outlined in the following steps:

$$
\begin{aligned}
& a \sqrt{1-u^{2}}=-(b u+c) \\
& d^{2}\left(1-u^{2}\right)=b^{2} u^{2}+2 b c u+c^{2} \\
& \left(a^{2}+b^{2}\right) u^{2}+2 b c u+c^{2}-a^{2}=0
\end{aligned}
$$

which gives

$$
u=\frac{-b c \cdot a \sqrt{a^{2}+b^{2}-c^{2}}}{a^{2}+b^{2}} .
$$

Substituting $u$ back into equation (47) we see that there are two roots $\therefore_{1}^{*}$ and "." such that

$$
\begin{aligned}
& \sin c^{*}=\frac{-b c+a \sqrt{a^{2}+b^{2}-c^{2}}}{a^{2}+b^{2}} \\
& \cos \theta_{1}^{*}=\frac{-a c-b \sqrt{a^{2}+b^{2}-c^{2}}}{a^{2}+b^{2}} \\
& \sin n^{*}=\frac{-b c-a \sqrt{a^{2}+b^{2}-c^{2}}}{a^{2}+b^{2}} \\
& \cos \theta^{*}=\frac{-a c+b \sqrt{a^{2}+b^{2}-c^{2}}}{a^{2}+b^{2}} .
\end{aligned}
$$


The expressions for $a^{2}+b^{2}$ and $a^{2}+b^{2}-c^{2}$ can be simplified using equations (45), giving the vector equations

$$
\begin{aligned}
& a^{2}+b^{2}=R_{1}^{2}\left(r^{2}+r_{0}^{2}-2 r r_{0} \cos \left(\phi-\phi_{0}\right)\right)=R_{1}^{2}\left|\underset{\sim}{r}-\underline{r}_{0}\right|^{2} \\
& a^{2}+b^{2}-c^{2}=R_{1}^{2}\left|\underline{r}-\underline{\sim}_{0}\right|^{2}-r^{2} r_{0}^{2} \sin ^{2}\left(\phi-\phi_{0}\right)=R_{1}^{2}\left|\underline{r}-\underline{\sim}_{0}\right|^{2}-\left|\underline{r} \times{\underset{\sim}{0}}_{0}\right|^{2} .
\end{aligned}
$$

Therefore $g(\theta)$ has two roots $\theta_{1}^{*}$ and $\theta_{2}^{*}$ which satisfy equations $(48)-(51)$.

4.2.2. The expressions for $|d g(\theta) / d \theta|_{\theta=\theta_{1}^{*}}$ and $|d g(\theta) / d \theta|_{\theta=\theta_{2}^{*}}$

Next we want to evaluate $|\mathrm{dg}(\theta) / d \theta|_{\theta=\theta_{1}^{*}}$ and $|\mathrm{dg}(\theta) / d \theta|_{\theta=\theta_{2}^{*}}$ where the derivative of $g(\theta)$ [equation (44)] with respect to $\theta$ is

$$
\frac{d g(\theta)}{d \theta}=\frac{(-a \sin \theta+b \cos \theta) s^{1 / 2}-(a \cos \theta+b \sin \theta+c) d S^{1 / 2} / d \theta}{S} .
$$

lie know fro.n equation (47) that the expression $a \cos \theta+b \sin \theta+c$ is equal to zero iur $"=\theta_{1}^{*}$ and $3=\theta_{2}^{*}$. Therefore

$$
\left|\frac{d g(\theta)}{d \theta}\right|_{\theta=\theta_{3}^{*}}=\left|\frac{-a \sin \theta_{1}^{*}+b \cos \theta_{1}^{*}}{s^{1 / 2}\left(\theta_{1}^{*}\right)}\right| .
$$

Substituting the expression for $\sin \theta_{1}^{\star}$ and $\cos \theta_{1}^{\star}$ given in equation (48) and equation (49) and using the expression in equation (53), gives

$$
\left|\frac{d g(\theta)}{d \theta}\right|_{\theta=\theta_{1}^{*}}=\frac{\sqrt{a^{2}+b^{2}-c^{2}}}{s^{1 / 2}\left(\theta_{1}^{\star}\right)}=\frac{\sqrt{R_{1}^{2}\left|\underline{r}-\underline{r}_{0}\right|^{2}-\left|\underline{r} \times \underline{r}_{0}\right|^{2}}}{s^{1 / 2}\left(\theta_{1}^{*}\right)}
$$


Likewise for $\theta=e_{2}^{\star}$ we have

$$
\begin{aligned}
\left|\frac{d g(0)}{d \theta^{*}}\right|_{\theta_{0=0_{2}^{*}}} & =\left|\frac{-a \sin \theta_{2}^{\star}+b \cos \theta_{2}^{\star}}{s^{\Pi / 2}\left(0_{2}^{\star}\right)}\right| \\
& =\frac{\sqrt{a^{2}+b^{2}-z^{2}}}{s^{1 / 2}\left(\theta_{2}^{\star}\right)}=\frac{\sqrt{R_{1}^{2}\left|r-r_{0}\right|^{2}-\left|r \times r_{0}\right|^{2}}}{s^{1 / 2\left(\theta_{2}^{\star}\right)}}
\end{aligned}
$$

\subsubsection{The expressions for $s\left(\theta_{1}^{\star}\right)$ and $s\left(\theta_{2}^{*}\right)$}

The denominators in equation (54) and equation (55) are evaluated by substituting $\sin \theta_{j}^{*}$ and $\cos 0_{i}^{\star}$ given by equations (48)-(51) into the expression for $S$ given in equation (45). First, for $S\left(\theta_{1}^{\star}\right)$ we have

$$
\begin{aligned}
S\left(\theta_{1}^{*}\right) & =r^{2}+R_{1}^{i}+2 R_{1} r \cos \phi \cos 11_{1}^{*}-2 R_{1} r \sin \sin _{1}^{*} \\
& =\frac{\left(r^{2}+R_{1}^{2}\right)\left(a^{2}+b^{2}\right)-2 R_{1} c(x a-y b)-2 R_{1}(x b+y a) \sqrt{a^{2}+b^{2}-c^{2}}}{a^{2}+b^{2}}
\end{aligned}
$$

where the expressions,

$$
\begin{aligned}
2 R_{1} c(x a-y b) & =2 R_{1}\left(x_{0} y-x y_{0}\right)\left[x R_{1}\left(y-y_{0}\right)-y R_{1}\left(x-x_{0}\right)\right] \\
& =2 R_{1}^{2}\left|r_{0} \times \underset{\sim}{r}\right|^{2}
\end{aligned}
$$

and

$$
\begin{aligned}
2 R_{1}(x b+y a) \sqrt{a^{2}+b^{2}-c^{2}} & =2 R_{1}\left(x R_{1}\left(x-x_{0}\right)+y R_{1}\left(y-y_{0}\right)\right) \sqrt{a^{2}+b^{2}-c^{2}} \\
& =2 R_{1}^{2}\left[r \cdot\left(\sim_{\sim}-\underline{\sim}_{0}\right)\right] \sqrt{R_{1}^{2}\left|r-r_{0}\right|^{2}-\left|r \times r_{0}\right|^{2}} .
\end{aligned}
$$


$-30-$

Substituting these expressions along with equations (52) and (53) into equation (56) gives

$$
\begin{aligned}
& S\left(\theta_{1}^{*}\right)=\frac{\left.\left(r^{2}+R_{1}^{2}\right) R_{1}^{2}\left|\underline{r}-\underline{\sim}_{0}\right|^{2}-2 R_{1}^{2}\left|\underline{\sim}_{0} \times \underset{\sim}{r}\right|^{2}-2 R_{1}^{2}\left[\underline{\sim}_{(-2}-\underline{\sim}_{0}\right)\right] \sqrt{R_{1}^{2}\left|\underline{r}-\underline{\sim}_{0}\right|^{2}-\left|\underline{r} \times \underline{\sim}_{0}\right|^{2}}}{R_{1}^{2}\left|\underline{\sim}-\underline{\sim}_{0}\right|^{2}}
\end{aligned}
$$

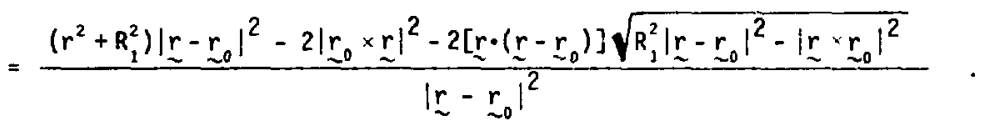

We can rewrite $S\left(\theta_{2}^{*}\right)$ as

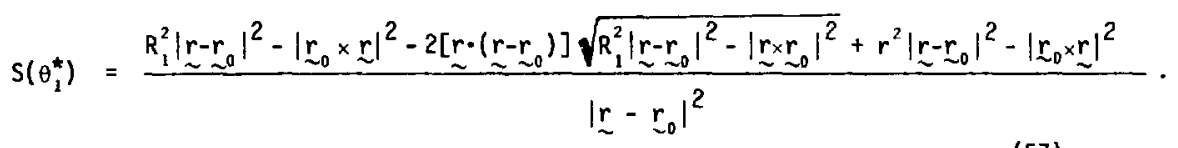

Since $\left[\underset{\sim}{r} \cdot(\underbrace{r-r_{0}}_{\sim})\right]^{2}=r^{2}\left|\underset{\sim}{r-r_{0}}\right|^{2}-\left|\sim_{0} \times \underset{\sim}{r}\right|^{2}$, the numerator in equation (57) is a perfect square and we can write $S\left(\theta_{1}^{*}\right)$ as

$$
S\left(\theta_{1}^{*}\right)=\frac{\left\{\sqrt{R_{1}^{2}\left|\underset{\sim}{r}-\sim_{0}\right|^{2}-\left|\underline{\sim}_{0} \times \underset{\sim}{r}\right|^{2}}-\underset{\sim}{r} \cdot\left(\underset{\sim}{r}-\underline{\sim}_{0}\right)\right\}^{?}}{\left|r-\underline{\sim}_{0}\right|^{2}} .
$$

For $S\left(\theta_{2}^{*}\right)$ we obtain

$$
\begin{aligned}
& S\left(\theta_{2}^{\star}\right)=r^{2}+R_{1}^{2}+2 R_{1} r \cos \phi \cos \theta_{2}^{\star}-2 R_{1} r \sin \phi \sin \theta_{2}^{\star} \\
& =\frac{\left(r^{2}+R_{1}^{2}\right)\left(a^{2}+b^{2}\right)-2 R_{1} c(a x-b y)+2 R_{1}(x b+y a) \sqrt{a^{2}+b^{2}-c^{2}}}{a^{2}+b^{2}}
\end{aligned}
$$

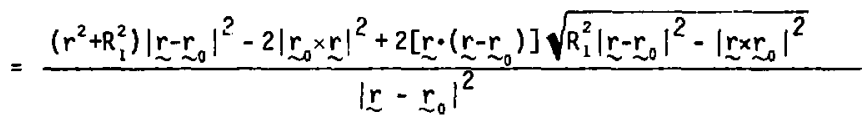

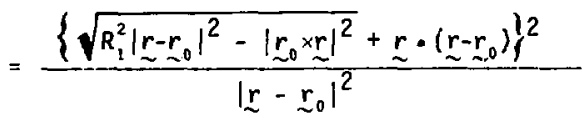


Substituting the expression for $5\left(\theta_{1}^{*}\right)$ into equation (54) gives the expression for $|d g(\theta) / d \theta|_{\theta=\theta_{1}^{\star}}$,

$$
\left|\frac{d g(\theta)}{d \theta}\right|_{0=\theta_{2}^{*}}=\frac{|\underset{r-r}{r}| \sqrt{R_{1}^{2}\left|r-r_{0}\right|^{2}-\left|r \times r_{0}\right|^{2}}}{\sqrt{R_{1}^{2}\left|r-\sim_{0}\right|^{2}-\left|\sim_{0} \times r\right|^{2}}-\underset{\sim}{r}\left(\underset{\sim}{r}-r_{0}\right)}
$$

and substituting the expression for $S\left(\theta_{2}^{*}\right)$ into equation (55) gives the expression for $|\mathrm{dg}(\theta) / \mathrm{d} \theta|_{\theta=\theta_{2}^{*}}$,

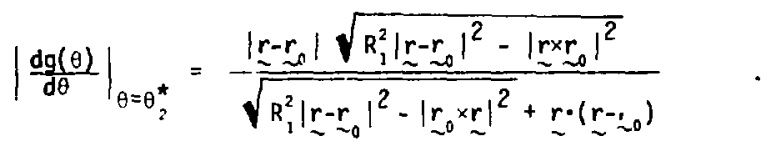

Notice that the expressions for these derivations differ only in the sign of the second term in the denominator. The denominator in equation $(60)$ would normally be expressed as the absolute value since it comes from the square root of a perfect square. However, if we assume that $R_{1}$ is greater than $r_{0}$ and $r$ then $\sqrt{R_{1}^{2}\left|r-r_{0}\right|^{2}-\left|\underline{r}_{0} \times r\right|^{2}}>\underline{r} \cdot\left(\underline{r-r_{0}}\right)=\sqrt{r^{2}\left|r-r_{0}\right|^{2}-\left|\sim_{0} \times r\right|^{2}}$ and the denominator expressed in equation $(60)$ is precisely equal to its absolute value. 


\subsubsection{Solution to the transformed adjoint transform}

We can now rewrite the adjoint transform given in equation (45) as

$p_{<}^{+}(r, \phi)=\int_{-\phi}^{\pi-\phi} \frac{R_{1}^{2} r \sin (\theta+\phi)\left[R_{1}+r \cos (\phi+\theta)\right]}{s^{3 / 2}(\theta)}\left\{\frac{\delta\left(0-\theta_{2}^{*}\right)}{\left|\frac{d g(0 !}{d \theta}\right|_{A=\theta_{1}^{*}}}+\frac{\delta\left(\theta-\theta_{2}^{*}\right)}{\left|\frac{d g(0)}{d \theta}\right|_{\theta=\theta_{2}^{*}}}\right\} d !$

Where the expressions for $|d g(\theta) / d \theta|_{\theta=\theta_{1}^{*}}$ and $|d g(0) / d \theta|_{\theta=\theta_{2}^{*}}$ are given by equations (54) and (55). Integrating aver $\theta$ the adjoint transform of the point source projection function can be expressed as

$p_{c}^{+}(r, \phi)=\sum_{i=1}^{2} \frac{R_{1}^{2} r \sin \left(\theta_{i}^{\star}+\phi\right)\left[R_{1}+r \cos \left(\phi+\theta_{i}^{\star}\right)\right]}{S\left(\theta_{i}^{\star}\right) \sqrt{R_{i}^{2}\left|r-r_{0}\right|^{2}-\left|r<r_{0}\right|^{2}}} D\left[\theta_{i}^{\star} \mid(-\phi, \pi-\phi)\right]$

where $\left.D\left[\theta_{i}^{*}\right)(-\phi, \pi-\phi)\right]=1$ if $\theta_{i}^{\star} \varepsilon(-\phi, \pi-\phi)$ and 0 otherwise. From the information we have about $\theta_{1}^{\star}$ and $\theta_{2}^{\star}$ given in equations (48)-(51), it is not imediately obvious whether $\theta_{1}^{*}$ and $\theta_{2}^{*}$ are both in the interval $(-\phi, \pi-\phi)$ or just one or neither. Since the terms of equation (63) must be positive for any $\theta \varepsilon(-\phi, \pi-\phi)$, we can determine under what conditions the roots $\theta_{1}^{*}$ and $\theta_{2}^{*}$ will be in the interval $(-\phi, \pi-\phi)$ by evaluating equation (63). It turns out that oniy one of the two roots will be in the interval $(-\phi, \pi-\phi)$ for any projected point source.

For $\mathbf{j}=1$, we have

$$
\begin{aligned}
r \sin \left(\theta_{1}^{\star}+\phi\right) & =r \cos \phi \sin \theta_{1}^{\star}+r \sin \phi \cos \theta_{1}^{\star} \\
& =r \cos \phi\left(\frac{-b c+a \sqrt{a^{2}+b^{2}-c^{2}}}{a^{2}+b^{2}}\right)+r \sin \phi\left(\frac{-a c-b \sqrt{a^{2}+b^{2}-c^{2}}}{a^{2}+b^{2}}\right) \\
& =\frac{-(x b+y a) c+(x a-y b) \sqrt{a^{2}+b^{2}-c^{2}}}{a^{2}+b^{2}}
\end{aligned}
$$


$-33-$

Substituting the values for $a, b, c, a^{2}+b^{2}$, and $\sqrt{a^{2}+b^{2}-c^{2}}$ from equations (45). (52) and (53) gives

$$
\begin{aligned}
& \operatorname{rsin}\left(0_{1}^{*}+1\right)=\frac{-R_{1}\left(x\left(x-x_{0}\right)+y\left(y-y_{0}\right)\right)\left(x_{0} y-x_{0} y_{0}\right)+R_{1}\left(x\left(y-y_{0}\right)-y\left(x-x_{0}\right)\right) \sqrt{R_{1}^{2}\left|r-r_{0}\right|^{2}-\mid r k r} \underbrace{2}_{0}}{R_{1}^{2}\left|r-r_{0}\right|^{2}} \\
& =\frac{-r \cdot\left(\underline{r-r_{0}}\right)\left(x_{0} y-x y_{0}\right)+\left(x_{0} y-x y_{0}\right) \sqrt{R_{1}^{2}\left|r-\underline{r}_{0}\right|^{2}-\left|\underline{x_{x}} \underline{r}_{0}\right|^{2}}}{R_{1}\left|\underline{r}-\underline{r}_{0}\right|^{2}} \\
& =\left(x_{0} y-x y_{0}\right) \frac{-\underline{r} \cdot\left(\underline{r}-\underline{r}_{0}\right)+\sqrt{R_{1}^{2}\left|\underline{r}-\underline{-}_{0}\right|^{2}-\left|\underline{r} \times \underline{r}_{0}\right|^{2}}}{R_{1}\left|\underline{r}-\underline{r}_{0}\right|^{2}}
\end{aligned}
$$

Likewise for $\operatorname{rcos}\left(\theta_{1}^{\star}+\phi\right)$, we have

$$
\begin{aligned}
& \operatorname{rcos}\left(11_{1}^{*}+\phi\right)=r \cos \phi_{\cos 0_{1}^{*}}-r \sin \phi \sin \theta_{1}^{*} \\
& =x\left(\frac{-a c-b \sqrt{a^{2}+b^{2}-c^{2}}}{a^{2}+b^{2}}\right)-y\left(\frac{-b c+a \sqrt{a^{2}+b^{2}-c^{2}}}{a^{2}+b^{2}}\right) \\
& =\frac{-(x a-y b) c-(x b+y a) \sqrt{a^{2}+b^{2}-c^{2}}}{a^{2}+b^{2}} \\
& =\frac{-R_{1}\left(x\left(y-y_{0}\right)-y\left(x-x_{0}\right)\right)\left(x_{0} y-x_{0}\right)-R_{1}\left(x\left(x-x_{0}\right)+y\left(y-y_{0}\right)\right) \sqrt{R_{1}^{2}\left|r-r_{0}\right|^{2}-\left|r x r_{0}\right|^{2}}}{R_{1}^{2}\left|r-\underline{\sim}_{0}\right|^{2}} \\
& =\frac{-\left|\underline{r}_{0} \times r\right|^{2}-\underset{\sim}{r} \cdot\left(\underset{\sim}{r-r_{0}}\right) \sqrt{R_{1}^{2}\left|r-r_{0}\right|^{2}-\left|\underline{r} \times \sim_{0}\right|^{2}}}{R_{1}\left|r-\underline{r}_{0}\right|^{2}}
\end{aligned}
$$

If we express $r \sin \left(\theta_{1}^{*}+\phi\right)$ and $r \cos \left(\theta_{1}^{\star}+\phi\right)$ as 


$$
\begin{aligned}
& r \sin \left(\theta_{1}^{*}+\phi\right)=\left(x_{0} y-x y_{0}\right) \frac{A+S^{*}}{R_{1}\left|\underline{r}-\underline{r}_{0}\right|^{2}} \\
& r \cos \left(\theta_{1}^{*}+\phi\right)=\frac{B+A S^{-}}{R_{1}\left|\underline{r}-\underline{\sim}_{0}\right|^{2}}
\end{aligned}
$$

where

$$
\begin{aligned}
& A=\underset{\sim}{r} \cdot\left(\underset{\sim}{r}-\sim_{0}^{r_{0}}\right) \\
& B=-\left|\underline{r} \times \underset{\sim}{r_{0}}\right|^{2} \\
& S=\sqrt{R_{1}^{2}\left|r-\sim_{0}\right|^{2}-\sim_{\sim}^{r} \times\left.\sim_{0}^{r}\right|^{2}},
\end{aligned},
$$

then the first term in equation (63) gives

$$
\begin{aligned}
& \frac{\left.R_{1}^{2} r \operatorname{sini} \theta_{1}^{*}+\phi\right)\left[R_{1}+r \cos \left(\theta_{1}^{*}+\phi\right)\right]}{S\left(\theta_{1}^{*}\right) \sqrt{R_{1}^{2}\left|r-r_{0}\right|^{2}-\left|r \times r_{g}\right|^{2}}} \\
& =\frac{R_{1}^{2}\left[R_{1} r \sin \left(\theta_{1}^{*}+\phi\right)+r \sin \left(\theta_{1}^{*}+\phi\right) r \cos \left(\theta_{1}^{*}+\phi\right)\right]}{S\left(e_{1}^{*}\right) \sqrt{R_{1}^{2}\left|r-r_{2}\right|^{2}-\left|r_{\sim} \times r_{0}\right|^{2}}} \\
& =\frac{R_{1}^{2}\left[R_{1}\left(x_{0} y-x y_{0}\right) \frac{A+S^{-}}{R_{1}\left|\underline{r}-\underline{\sim}_{0}\right|^{2}}+\left(x_{0} y-x y_{0}\right)\left(\frac{A+S^{-}}{R_{1}\left|\underline{r}-r_{0}\right|^{2}}\right)\left(\frac{B+A S^{-}}{R_{1}\left|\underline{r}-r_{0}\right|^{2}}\right)\right]}{S\left(\theta_{1}^{*}\right) \cdot S^{-}} \\
& =\frac{R_{1}^{2}\left(x_{0} y-x y_{0}\right)\left[R_{1}^{2}\left|\underset{\sim}{r}-\sim_{0}\right|^{2}\left(A+S^{\prime}\right)+\left(A+S^{\prime}\right)\left(B+A S^{\prime}\right)\right]}{S\left(\theta_{1}^{*}\right) \cdot S^{\prime} R_{1}^{2}\left|\underset{\sim}{r}-\sim_{0}\right|^{4}}
\end{aligned}
$$

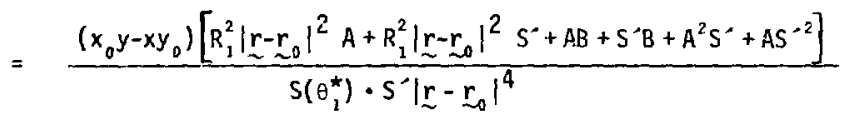




$$
\begin{aligned}
& =\frac{\left(x_{0} y-x y_{0}\right)\left[R_{1}^{2}\left|r-r_{0}\right|^{2} A+A B+A S^{-2}+\left(R_{1}^{2}\left|r-r_{0}\right|+B+A^{2}\right) S^{-}\right]}{S\left(\theta_{1}^{\star}\right) \cdot S^{*}\left|r-r_{0}\right|^{4}} \\
& =\frac{\left(x_{0} y-x y_{0}\right)\left[\left(R_{1}^{2} \mid \underset{\sim}{\left.\left.r-\left.r_{0}\right|^{2}+B\right) A+A S^{-2}+\left(S^{-2}+A^{2}\right) S^{-}\right]}\right.\right.}{S\left(\theta_{2}^{*}\right) S^{\prime} \mid \underset{\sim}{r-\left.r_{0}\right|^{4}}} \\
& =\frac{\left(x_{0} y-x y_{0}\right)\left[S^{-2} A+A S^{-2}+\left(S^{-2}+A^{2}\right) S^{\prime}\right]}{S\left(\theta_{2}^{\star}\right) S^{\prime}\left|r-r_{0}\right|^{4}} \\
& =\left(x_{0} y-x y_{0}\right) \frac{\left[S^{-2}+2 A S^{-}+A^{2}\right]}{S\left(\theta_{1}^{*}\right)\left|r_{-}-r_{0}\right|^{4}} \\
& =\left(x_{0} y-x y_{0}\right) \frac{\left(S^{-}+A\right)^{2}}{S\left(\theta_{1}^{*}\right) \mid \underline{L-\left.r_{-0}\right|^{4}}}
\end{aligned}
$$

If we substitute $S\left(\theta_{1}^{\star}\right)$ from equation $(58)$ into the above equation, we have

$$
\begin{aligned}
& \frac{R_{1} r \sin \left(\theta_{1}^{*}+\phi\right)\left[R_{1}+r \cos \left(\theta_{1}^{*}+\phi\right)\right]}{S\left(\theta_{1}^{*}\right) \sqrt{R_{1}^{2}\left|r-r_{0}\right|^{2}-\left|r \times r_{0}\right|^{2}}} \\
& =\left(x_{0} y-x y_{0}\right) \frac{(S+A)^{2}\left|\underline{\sim}-\sim_{0}\right|^{2}}{\left\{\sqrt{R_{1}^{2}\left|r-r_{0}\right|^{2}-\left|r_{0} \times r\right|^{2}}-\underset{\sim}{r}\left(\underset{\sim}{\left.\left.r-r_{0}\right)\right\}^{2}\left|r-r_{0}\right|^{4}}\right.\right.} \\
& =\frac{\left(x_{0} y-x y_{0}\right)}{\left|r-r_{0}\right|^{2}} \frac{(s-A)^{2}}{(s-A)^{2}}=\frac{\left(x_{0} y-x y_{0}\right)}{\left|r-r_{0}\right|^{2}}
\end{aligned}
$$

Equation (67) is the expression for the first term in equation (63) and immediateiy one sees that this is positive only if $\left(x_{0} y-x y_{0}\right)>0$. Therefore $\theta_{1}^{*}$ is in the interval $(-\phi, \pi-\phi)$ only if $\left(x_{0} y-x y_{0}\right)>0$. 
For $i=2$, we have

$$
\begin{aligned}
& r \sin \left(\theta_{2}^{\star}+\phi\right)=x \frac{-b c-a \sqrt{a^{2}+b^{2}-c^{2}}}{a^{2}+b^{2}}+y \frac{-a c+b \sqrt{a^{2}+b^{2}-c^{2}}}{a^{2}+b^{2}} \\
& =\frac{-(x b+y a) c+(-x a+y b) \sqrt{a^{2}+b^{2}-c^{2}}}{a^{2}+b^{2}} \\
& =\frac{-R_{1}\left(x\left(x-x_{0}\right)+y\left(y-y_{0}\right)\right)\left(x_{0} y-x y_{0}\right)+R_{1}\left(-x\left(y-y_{0}\right)+y\left(x-x_{0}\right)\right) \sqrt{R_{1}^{2}\left|\underline{-}-\underline{r}_{0}\right|^{2}-|\underbrace{r}_{0} \times r_{0}|^{2}}}{R_{1}^{2}\left|r-\underline{r}_{0}\right|^{2}}
\end{aligned}
$$

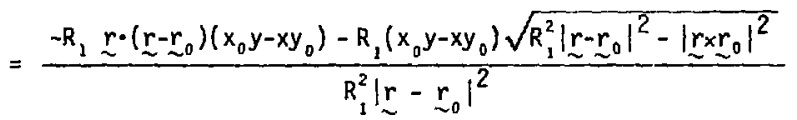

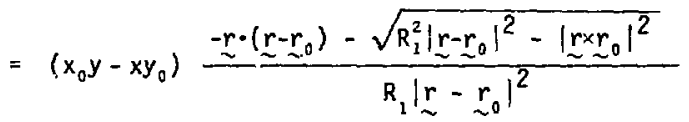

$$
\begin{aligned}
& \operatorname{rcos}\left(\theta_{2}^{*}+\phi\right)=x \frac{-a c+b \sqrt{a^{2}+b^{2}-c^{2}}}{a^{2}+b^{2}}-y \frac{-b c-a \sqrt{a^{2}+b^{2}-c^{2}}}{a^{2}+b^{2}} \\
& =\frac{(-x a+y b) c+(x b+y a) \sqrt{a^{2}+b^{2}-c^{2}}}{a^{2}+b^{2}} \\
& =\frac{R_{1}\left(-x\left(y-y_{0}\right)+y\left(x-x_{0}\right)\right)\left(x_{0} y-x_{0}\right)+R_{1}\left(x\left(x-x_{0}\right)+y\left(y-y_{0}\right)\right) \sqrt{R_{1}^{2}\left|r-\sim_{0}\right|^{2}-\left|r_{2} \times \underline{r}_{0}\right|^{2}}}{R_{1}^{2}\left|r-\sim_{0}^{r_{0}}\right|^{2}}
\end{aligned}
$$

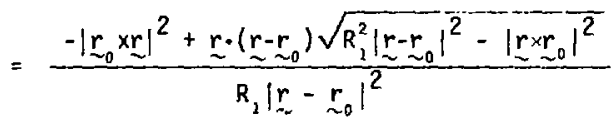


If we express $r \sin \left(\theta_{2}^{\star}+\phi\right)$ and $r \cos \left(\theta_{2}^{*}+\phi\right)$ as

$$
\begin{aligned}
& r \sin \left(0_{2}^{*}+\phi\right)=\left(x_{0} y-x_{0}\right) \frac{A-S^{\circ}}{R_{1}\left|\underline{r}-\underline{r}_{0}\right|^{2}} \\
& r \cos \left(0_{2}^{\star}+\phi\right)=\frac{B-A S^{\circ}}{R_{1}\left|\underline{r}-\underline{r}_{0}\right|^{2}}
\end{aligned}
$$

where $A, B$, and $S^{-}$are defined in equation (66), then the second fraction of equation (63) becomes

$$
\begin{aligned}
& \frac{R_{1}^{2} r \sin \left(\theta_{2}^{\star}+\phi\right)\left[R_{1}+r \cos \left(\phi+\theta_{2}^{*}\right)\right]}{S\left(\theta_{2}^{\star} ; \sqrt{R_{1}^{2}\left|r-r_{0}\right|^{2}-\left|r \times r_{0}\right|^{2}}\right.} \\
& =\frac{R_{1}^{2}\left[R_{1} r \sin \left(\theta_{2}^{*}+\phi\right)+r \sin \left(\theta_{2}^{*}+\phi\right) r \cos \left(\phi^{+} \theta_{2}^{*}\right)\right]}{S\left(\theta_{2}^{*}\right) \sqrt{R_{1}^{2}|r-r-0|^{2}-|\underbrace{r \times r}_{\sim 0}|^{2}}}
\end{aligned}
$$

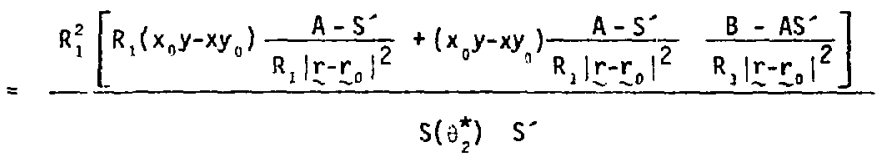

$$
\begin{aligned}
& =\frac{R_{1}^{2}\left(x_{0} y-x y_{0}\right)\left[R_{1}^{2}\left|r-r_{0}\right|^{2}\left(A-S^{\prime}\right)+\left(A-S^{\prime}\right)\left(B-A S^{\prime}\right)\right]}{S\left(\theta_{2}^{*}\right) S^{\prime} R_{1}^{2}\left|r-r_{0}\right|^{4}} \\
& =\frac{\left(x_{0} y-x y_{0}\right)\left[R_{1}^{2}\left|\underline{r}-\underline{r}_{0}\right|^{2} A-R_{1}^{2}\left|\underline{r-r_{0}}\right|^{2} S^{\prime}+A B-S^{-B} B-A^{2} S^{\prime}+A S^{-2}\right]}{S\left(\theta_{2}^{*}\right) S^{0} \mid \underline{r-\left.r_{0}\right|^{4}}} \\
& =\frac{\left\langle x_{0} y-x y_{0}\right)\left[R_{1}^{2}\left|r-r_{0}\right|^{2} A+A B+A S^{\prime 2}-\left(R_{1}^{2}\left|r-r_{0}\right|^{2}+B+A^{2}\right) S^{*}\right]}{S\left(\theta_{2}^{*}\right) S^{*}\left|\underline{\sim}-\sim_{0}\right|^{4}}
\end{aligned}
$$




$$
\begin{aligned}
& =\frac{\left(x_{0} y-x y_{0}\right)\left[2 S^{-2}-\left(S^{-2}+A^{2}\right) S^{\prime}\right]}{S\left(\theta_{2}^{*}\right) S^{*} \mid \underline{r}-\underline{r}_{0} i^{4}} \\
& =\frac{\left(x_{0} y-x y_{0}\right) S^{\circ}\left[2 S^{*}-S^{-2}-A^{2}\right]}{S\left(\theta_{2}^{*}\right) S^{-}\left|\underline{r}-\underline{r}_{0}\right|^{4}} \\
& =\frac{-\left(x_{0} y-x y_{0}\right)\left(S^{*}-A\right)^{2}}{S\left(\theta_{2}^{*}\right)\left|r-\underline{r}_{0}\right|^{4}}
\end{aligned}
$$

If we substitute $S\left(\theta_{2}^{*}\right)$ from equation (59) into the above equation, we get

$$
\begin{aligned}
& \frac{R_{1} r \sin \left(\theta_{2}+\phi\right)\left[R_{1}+r \cos \left(\theta_{2}^{*}+\phi\right)\right]}{s\left(\theta_{2}^{*}\right) \sqrt{R_{1}^{2}\left|r-r_{0}\right|^{2}-\left|r \times r_{0}\right|^{2}}}
\end{aligned}
$$

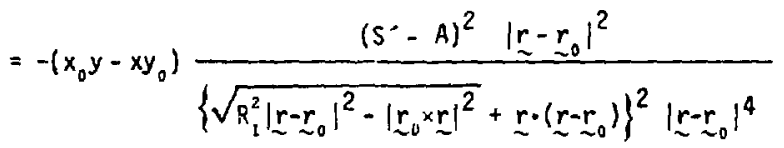

$$
\begin{aligned}
& =\frac{-\left(x_{0} y-x y_{0}\right)}{\left|r-\underline{\sim}_{0}\right|^{2}} \frac{(s-A)^{2}}{(s-A)^{2}}=\frac{-\left(x_{0} y-x y_{0}\right)}{\left|r-\underline{\sim}_{0}\right|^{2}}
\end{aligned}
$$

From equation (70) we see that the second term in equation (63) is positive only if $\left(x_{0} y-x y_{0}\right)<0$. Therefore $\theta_{2}^{*}$ is in the interval $(-\phi, \pi-\phi)$ only if $\left(x_{0} y-x y_{0}\right)<0$.

Comparing equations (67) and (70), we see that conditions for both $\theta_{1}^{*}$ and $\theta_{2}^{*}$ being in the interval $(-\phi, \pi-\phi)$ are mutually exclusive. Therefore using the results from equations (67) and (70) we can express the adjoint transform of a projected point source for the fan beam geometry given in equation (63) as 
$-39-$

$$
p_{<}^{+}(r, \phi)=\left\{\begin{array}{cc}
\frac{\left(x_{0} y-x y_{0}\right)}{\left|r-r_{0}\right|^{2}} & \text { if }\left(x_{0} y-x y_{0}\right)>0 \\
\frac{-\left(x_{0} y-x y_{0}\right)}{\left|r-r_{0}\right|^{2}} & \text { if }\left(x_{0} y-x y_{0}\right)<0
\end{array}\right.
$$

The expression $x_{0} y-x y_{0}$ represents the $z$-component of the vector cross product $r_{0} \times r$, therefore we can express $p_{<}^{+}(r, \phi)$ as

$$
P_{<}^{+}(r, \phi)=\frac{\left|r_{0} \times \underline{r}\right|}{\left|\underline{r}-\underline{r}_{0}\right|^{2}}
$$

4.3 Adjoint Transform of a Point Source Projection Function for

Parallel Beam Geometry

The adjoint transform of a point source projection function given by equation (15) for parallel beam geometry is equivalent tu the result obtained for the fan beam. If we substitute equation (15) into the expression for the adjoint transform given by equation (36) we have

$$
\mathbf{p}_{=}^{+}(r, \phi)=\int_{0}^{11} \delta\left(r \sin \left(\theta^{-}+\phi\right)-r_{0} \sin \left(\phi_{0}+\theta^{-}\right)\right)\left|r \sin \left(\theta^{+}+\phi\right)\right| d \theta^{\circ} \text {. }
$$

Aga in, in order to evaluate the integral we need to determine the zeros of

$$
f\left(\theta^{-}\right)=r \sin \left(\theta^{\circ}+\phi\right)-r_{0} \sin \left(\phi_{0}+\theta^{-}\right)
$$

which are $\theta_{1}^{\star}$ and $\theta_{2}^{\star}$ such that

$$
\sin \theta_{1}^{*}=\frac{r_{0} \sin \phi_{0}-r \sin \phi}{\left|\underline{r}_{0}-\underline{r}\right|}=\frac{y_{0}-y}{\left|\underline{r}_{0}-\underline{r}\right|}
$$




$$
\cos _{1}^{*}=\frac{r \cos -r_{0} \cos t_{0}}{\left|r_{0}-r\right|}=\frac{x-x_{0}}{\mid \underline{r}-\underline{-r \mid}}
$$

and

$$
\begin{aligned}
& \sin _{2}^{*}=\frac{r \sin \phi-r_{0} \sin \theta_{0}}{\left|r_{0}-r\right|}=\frac{y-y_{0}}{\left|\underline{r}_{0}-\underline{r}\right|} \\
& \cos 0_{2}^{\star}=\frac{r_{0} \cos \phi_{0}-r \cos \phi}{\left|r_{0}-r\right|}=\frac{x_{0}-x}{\left|r_{0}-r\right|}
\end{aligned}
$$

The derivative of $f$ with resoect to $\theta^{*}$ evaluated at $\theta_{1}^{*}$ and $A_{2}^{*}$ aives

$$
\left|\frac{d f\left(\theta^{\circ}\right)}{d \theta^{*}}\right|_{\theta^{\circ}=\theta_{1}^{*}, \theta_{2}^{*}}=\mid r_{-}-\underline{r}
$$

Therefore equation (72) can be expressed as

$$
p_{=}^{+}(r, \phi)=\int_{0}^{\pi} \frac{\left|r \sin \theta^{-} \cos \phi+r \sin \phi \cos \theta^{-}\right|}{\mid \underline{\sim}_{0}-\underline{r} !}\left[\delta\left(\theta^{-}-\theta_{1}^{*}\right)+\delta\left(\theta^{*}-\theta_{z}^{*}\right)\right] d \theta^{\prime} .
$$

Since the roots are $180^{\circ}$ out of phase the adjoint transform can be expresseo as

$$
p_{=}^{+}(r, \phi)=\frac{\left|y x_{0}-x y_{0}\right|}{\left|r-\sim_{0}\right|^{2}}
$$

Using the expression $\underline{\sim}_{0} \times \underset{r}{r}=y x_{0}-x y_{0}$, we can express the adjoint transform as

$$
p_{=}^{+}(r, \phi)=\frac{|\underline{\sim} \times \underset{\sim}{r}|}{\left|\underline{r}-\underline{r}_{0}\right|^{2}}
$$

Therefore the adjoint transform of a point source projection function for the parallel beam geometry given in equation (73) is identical to the result given in equation (71) for the far beam geometry. 


\subsection{The Back-Projection of a Fan Beam Point Source}

\section{Projection Function}

We have aiready seen the definition of the fan beam back-projection operator given in equation (4)). Notice that the adjoint operator given in equation (34) gives a weighted integral of the projection function $p_{<}\left(\xi^{*}, \theta\right)$ whereas the fan beam back-projection operator defined as

$$
b_{s}(r, \phi)=\int_{0}^{2 \pi} p_{<}\left(\xi^{*}, \theta\right) d \theta
$$

where $\xi^{\star}$ is given by equation (28), is an unweighted integral of the projection function. If we substitute the equation for the fan beam point source projection function: given in equation (14) into equation (41), then

$$
\left.b_{<}(r, \phi)=\int_{D}^{2 \pi} \varepsilon ! R_{1} \sin \xi * / R_{2}-r_{0} \sin \left(\phi_{0}+\theta-\xi^{*} / R_{2}\right)\right) d \theta \text {. }
$$

The back-projection function can be rewritten as

$$
b_{<}(r, \phi)=\int_{0}^{2 \pi} \frac{\delta\left(\theta-\theta_{1}^{*}\right)}{\left|\frac{d g(\theta)}{d \theta}\right|_{\theta=\theta_{2}^{*}}}+\frac{\delta\left(\theta-\theta_{2}^{*}\right)}{\left|\frac{d g(\theta)}{d \theta}\right|_{\theta=\theta_{2}^{*}}} d \theta
$$

where $\theta_{1}^{*}$ and $\theta_{2}^{*}$ satisfy equations (48)-(51) and $|d g(\theta) / d \theta|_{\theta=\theta_{2}^{*}}$ and $|d g(\theta) / d \theta|_{\theta=e_{2}^{*}}$ are given by equations $(60)$ and (61). Substituting these equations into equation (74) and integrating over $\theta$ gives

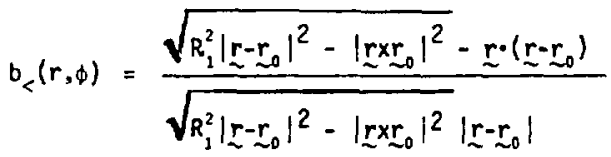

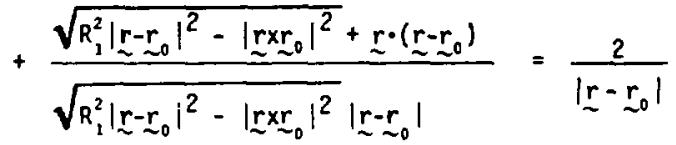




\subsection{The Back-Projection of a Parallel Beam Point Source}

\section{Projection Function}

The equation for the parallel beam back-projection operator is given in equation (39),

$$
b_{=}(r, \phi)=\int_{0}^{\pi} p_{=}\left(r \sin \left(\phi+\theta^{\prime}\right), \theta^{-}\right) d \theta^{-} .
$$

Substituting the equation for the parallel beam point source projection function given in equation (15) into equation (39) gives

$$
b_{=}(r, \phi)=\int_{0}^{\pi} \delta\left(r \sin \left(\phi+\theta^{-}\right)-r_{0} \sin \left(\phi_{0}+\theta^{-}\right)\right) d \theta^{-} .
$$

We can express $b_{=}(r, \phi)$ as

$$
b_{=}(r, \phi)=\int_{0}^{\pi} \frac{\delta\left(\theta^{*}-\theta_{2}^{*}\right)}{\left|\frac{d f\left(\theta^{-}\right)}{d \theta^{-}}\right|_{\theta^{*}=\theta_{1}^{*}}}+\frac{\delta\left(\theta^{*}-\theta_{2}^{\star}\right)}{\left|\frac{d f\left(\theta^{-}\right)}{d \theta^{*}}\right|_{\theta^{*}=\theta_{2}}} d \theta^{-}
$$

where $\theta_{2}^{*}$ and $\theta_{2}^{\star}$ are the roats of

$$
f(\theta)=r \sin \left(\phi+\theta^{-}\right)-r_{0} \sin \left(\phi_{0}+\theta^{-}\right)
$$

given in Section 4.4 and

$$
\left|\frac{d r^{-}\left(\theta^{\circ}\right)}{d \theta^{-}}\right|_{\theta^{*}=\theta_{1}^{\star}, \theta_{2}^{*}}=\left|\underline{\sim}_{0}-\underset{r}{r}\right|
$$

Since $\theta_{1}^{*}$ and $\theta_{2}^{*}$ are $180^{\circ}$ out of phase, then the result for the back-projection of a point source projection function is

$$
b_{=}(r, \phi)=\frac{1}{|r-\underset{\sim 0}{r}|} \text {. }
$$

The back-projection result for the parallel beam geometry differ from the result for a fan beam geometry only in a factor of 2 . 


\subsection{DISCUSSION}

The adjoint and back-projection operators for the fan beam and parallel beam geometries are summarized in Fig. 6. These operators are members of a class of operators which give the superposition of the projection line integrals weighted by a particular kernel which for the simple back-projection is equal to the identity function. The back-projection operator for the parallel beam geometry requires the summation of line integrals over a range of $180^{\circ}$ whereas the fan beam back-projection operator requires the summation of $l$ ine integrals over a range of $360^{\circ}$. Therefore, the fan beam geometry requires a double sampling of projection data which is reflected in the $2 /\left|r-\underline{\sim}_{0}\right|$ response for the back-projection of a point source projection function as opposed to a $1 /\left|\underline{r}-\underline{\sim}_{0}\right|$ response for the parallel beam backprojection operator.

The results of the fan beam back-projection operation for various point source orientations and rotations of $180^{\circ}$ and $360^{\circ}$ are shown in Fig. 7 . The projection data represent a sampling at $1^{\circ}$ increments. The middle column of figures are images of the back-projection multiplied by $|\underset{\sim}{r}-\underset{\sim}{r}|$ which has uniform gray only if the back-projection is proportional to $1 / \mid \underset{\sim}{r-r_{0} \mid}$. The back-projection operation for a rotation of $360^{\circ}$ is approximately proportional to $1 /\left|\underset{\sim}{r}-\underline{r}_{0}\right|$, but due to the digitalization, the image of $\left|\underset{r}{r} \underline{r}_{0}\right| \cdot B(r, \phi)$ represents a 10\% variation between the maximum and minimum values. The contrast for the case of $360^{\circ}$ rotation has been turned up to illustrate this variation.

The results shown in Fig. 7 for $2180^{\circ}$ sampling can be expressed explicitly as 
PARALLEL BEAM

$$
\begin{aligned}
& P_{-}\left(\xi^{\prime}, \theta^{\prime}\right)=\iint_{\mathbb{R}^{2}} f(x, y) \delta\left(\xi^{\prime}-x \sin \theta^{\prime}-y \cos \theta^{\prime}\right) d x d y \\
& p_{=}^{*}(r, \phi)=\int_{0}^{\pi} p\left(r \sin \left(\phi+\theta^{\prime}\right), \theta^{\prime}\right)\left|r \sin \left(\phi+\theta^{\prime}\right)\right| d \theta^{\prime}
\end{aligned}
$$

$$
b=r, \phi)=\int_{0}^{\pi} p\left(r \sin \left(\phi+\theta^{\prime}\right), \theta^{\prime}\right) d \theta^{\prime}
$$

For o point source at the point $\left(r_{0}, \phi_{0}\right)$

$$
\mathrm{p}\left(\xi^{\prime}, \theta^{\prime}\right)=\delta\left(\xi^{\prime}-r_{0} \sin \left(\phi_{0}+\theta^{\prime}\right)\right)
$$

$$
\begin{aligned}
& p_{s}^{+}(r, \phi)=\frac{\left|r_{0} \times r\right|}{\left|r-r_{0}\right|^{2}} \\
& b(r, \phi)=\frac{1}{\left|\underline{r}-I_{0}\right|}
\end{aligned}
$$

FAN BEAM

$$
\begin{aligned}
& p_{c}(\xi, \theta)=\iint_{\mathbb{R}^{2}} f(x, y) \delta\left(R_{1} \sin \xi / R_{2}-x \sin \left(\theta-\xi / R_{2}\right)-y \cos \left(\theta-\xi / R_{2}\right)\right) d x d y \\
& p_{c}^{*}(r, \phi)=\int_{-\phi}^{\pi-\phi} \frac{R_{1}^{2} p_{s}\left(\xi_{1}^{*}, \theta\right) r \sin (\phi+\theta)\left[R_{1}+r \cos (\phi+\theta)\right] d \theta}{\left[r^{2}+R_{1}^{2}+2 R_{1} r \cos (\phi+\theta)\right]^{3 / 2}} \\
& \text { where } \xi^{*}=R_{2} \tan ^{-1}\left[\frac{r \sin (\phi+\theta)}{R_{1}+r \cos (\phi+\theta)}\right] \\
& b_{c}(r, \phi)=\int_{0}^{2 \pi} R_{k}\left(\xi^{*}, \theta\right) d \theta
\end{aligned}
$$$$
p_{\varepsilon}(\xi, \theta)=\delta\left(R_{1} \sin \zeta / R_{2}-r_{0} \sin \left(\phi_{0}+\theta-\xi / R_{2}\right)\right)
$$$$
p_{c}(r, \phi)=\frac{|\underline{r} 0 \times x|}{\left|\underline{r}-I_{0}\right|^{2}}
$$$$
b_{6}(r, \phi)=\frac{2}{\left|\underline{r}-I_{0}\right|}
$$

$\times B L 768-9234$

Figure 6. Expressions for the projection, adjoint and back-projection operations for the fan beam and parallel beam geometry and the results for a point source with coordinates $\left(r_{0}, \phi_{0}\right)$. 
POINT SOURCE RESPONSE AS A FUNCTION OF POSITION

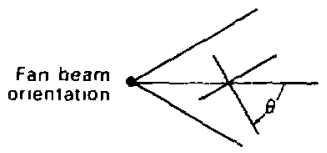

Rotated $180^{\circ}$

Back.Projection

Back-Projection. $\mid\left[-\mathfrak{t}_{0} \mid\right.$

Reconstruction
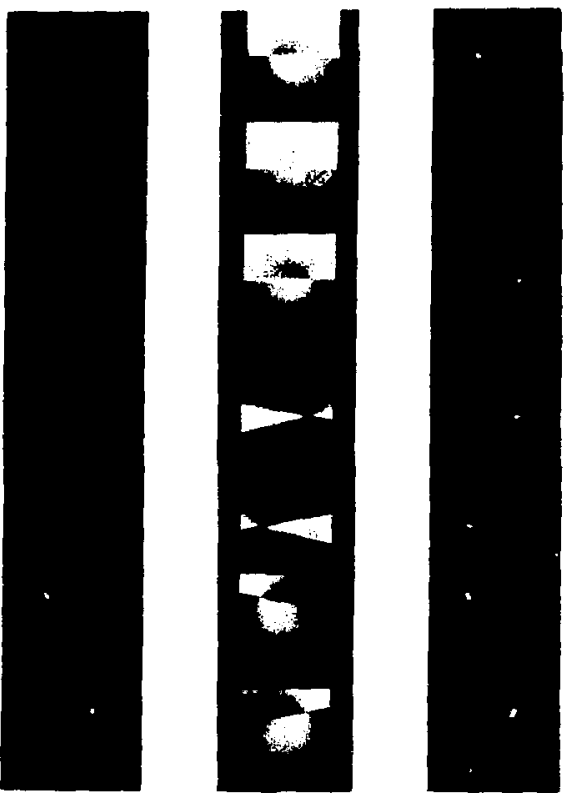

Rotated $360^{\circ}$
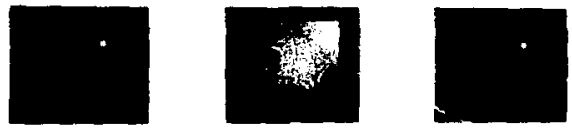

XBB 760-96́38

Figure 7. Comparison of the point source response as a function of position and rotation angle. The fan beam vertex coordinates are $R_{1}=80$ and $R_{2}=120$. 


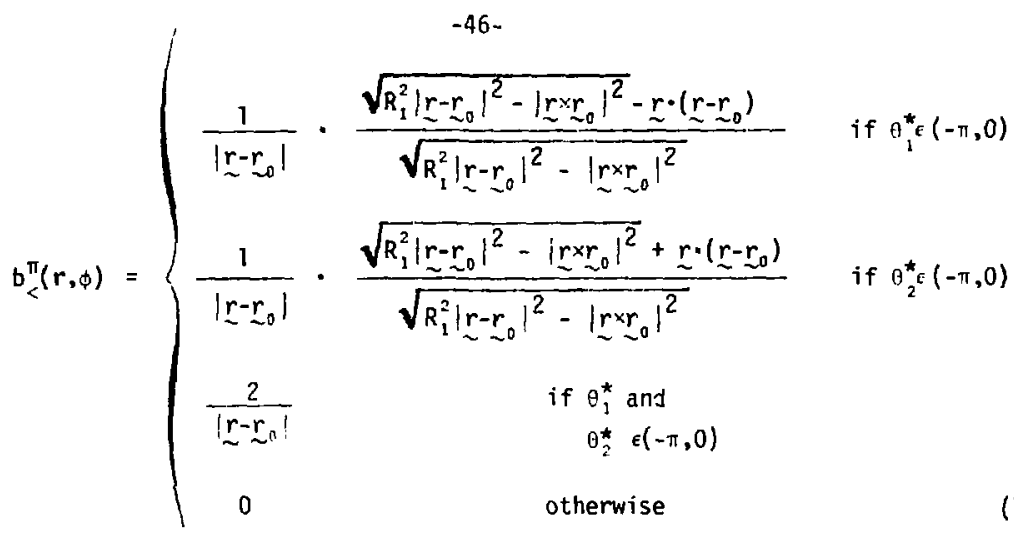

where $\theta_{1}^{*}$ and $\theta_{2}^{*}$ satisfy equations $(48)-(51)$. Equation (76) follows immediately from equation (74) where the limits of integration are taken to be from 0 to $-\pi$ instead of from 0 to $2 \pi$. A similar result was given by Peters [3] for the impulse response of the fan beam layergram. For a centrally positioned source $\left(r_{0}=0\right)$ we see from equations (48) and (50)

$$
\begin{aligned}
& \sin \theta_{1}^{*}=\frac{y}{\mid \underline{|r|}} \\
& \sin \theta_{2}^{*}=\frac{-y}{\mid \underline{|r|}} .
\end{aligned}
$$

Therefore for points in the lower half of the plane $\theta_{1}^{\star} E(-\pi, 0)$ and equation (76) reduces to

$$
|\underline{r}| b_{<}^{\pi}(r, \phi)=1-\frac{|r|}{R_{1}}
$$

For points in the upper half of the plane $\theta_{2}^{\star} \epsilon(-\pi, 0)$ and equation $(76)$ reduces to

$$
\underset{\sim}{\mid r} \mid b_{<}^{\pi}(r, \phi)=1+\frac{|\underline{\sim}|}{R_{1}}
$$


Therefore for a centrally positioned point source the upper and lower planes show a contrast about the abscissa as the result of the discontinuity at $y=0$.

Fiqures $B$ and 9 give a comparison of the results from filtering the pärallel beam and fan beam back-projection for two simulation phantoms. Artifacts are generated when the fan beam back-projection operation is integrated only over $180^{\circ}$. For an infinite number of projections, the metnod of filtering the back-projection for parallel beam geometry can be expressed as

$$
f(r, \phi)=\mathcal{F}_{2}^{-1}\left\{|R| \mathcal{J}_{2}(b=\{r, \phi)\}\right\}
$$

and for the fan beam geometry as

$$
f(r, \phi)=\frac{1}{2} \mathcal{G}_{2}^{-1}\left\{|R| \mathscr{T}_{2}\left(b_{<}(r, \phi)\right\}\right\} .
$$

However, the Parzen filter (Fig. 10) was used instead of the ramp filter in order to eliminate the ringing which occurs with a sharp cut-off. Other filters such as the Hamming, Hann, and Butterworth have been studied and give results comparable to that of the parzen filter. Of these filters, the Hann filter appears to give the best results.

Three key points are derived from the analysis presented:

1. There exists a fan beam back-projection operator which gives a result equal to $2 /\left|\underset{\sim}{r}-{\underset{\sim}{0}}_{0}\right|$ for a point source projection function.

2. This operator involves the integration of projection data taken over $360^{\circ}$ and not just $180^{\circ}$ which is sufficient for a parallel beam geometry. 


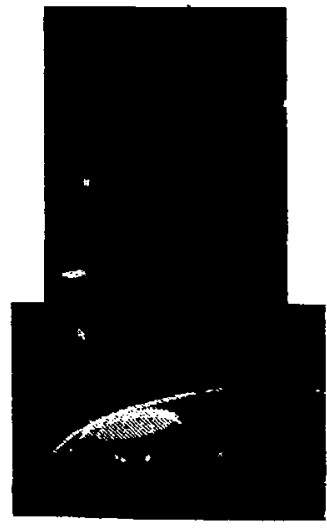

Original

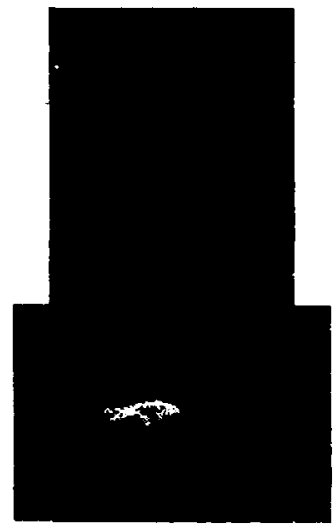

Porallel 28 Proj. $64^{\circ}$

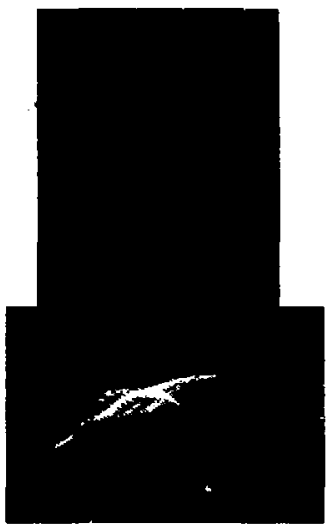

Fan Beam 28 Proj. $64^{\circ}$

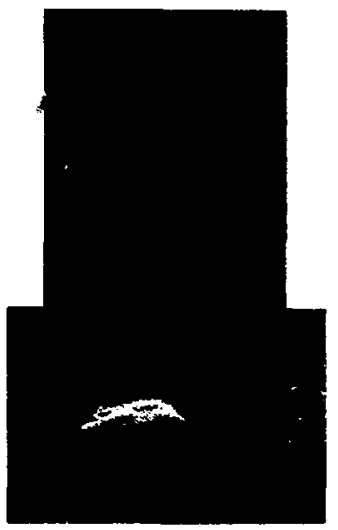

Fan Beam 56 Proj. $6.4^{\circ}$

XBB 763-2175

Figure 8. Comparison of results from filtering the back-projection foi parallel beam and fan team geometries. 


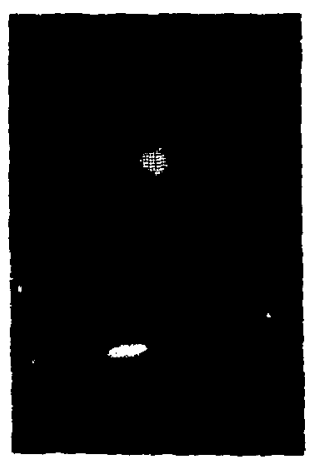

Original

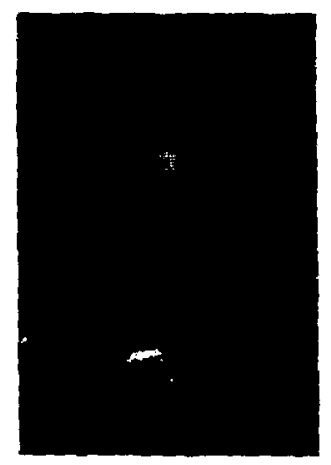

Parallel 90 Proj. $2^{\circ}$

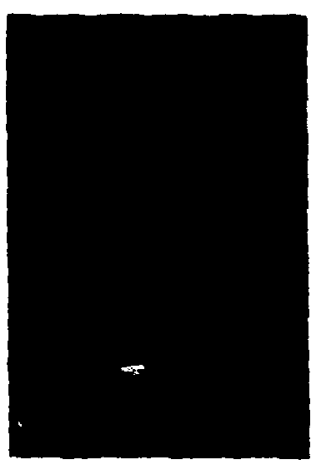

Fan Beam 90 Proj. $2^{\circ}$

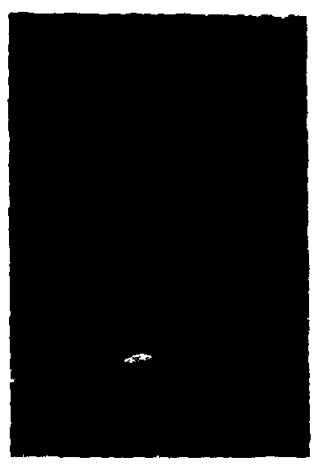

Fan Beam 180 Droj. $2^{\circ}$ XBB $762-1243$

Figure 9. Comparison of resuits from filtering the back-projection

\begin{abstract}
for parallel beam and fan beam geometries.
\end{abstract}




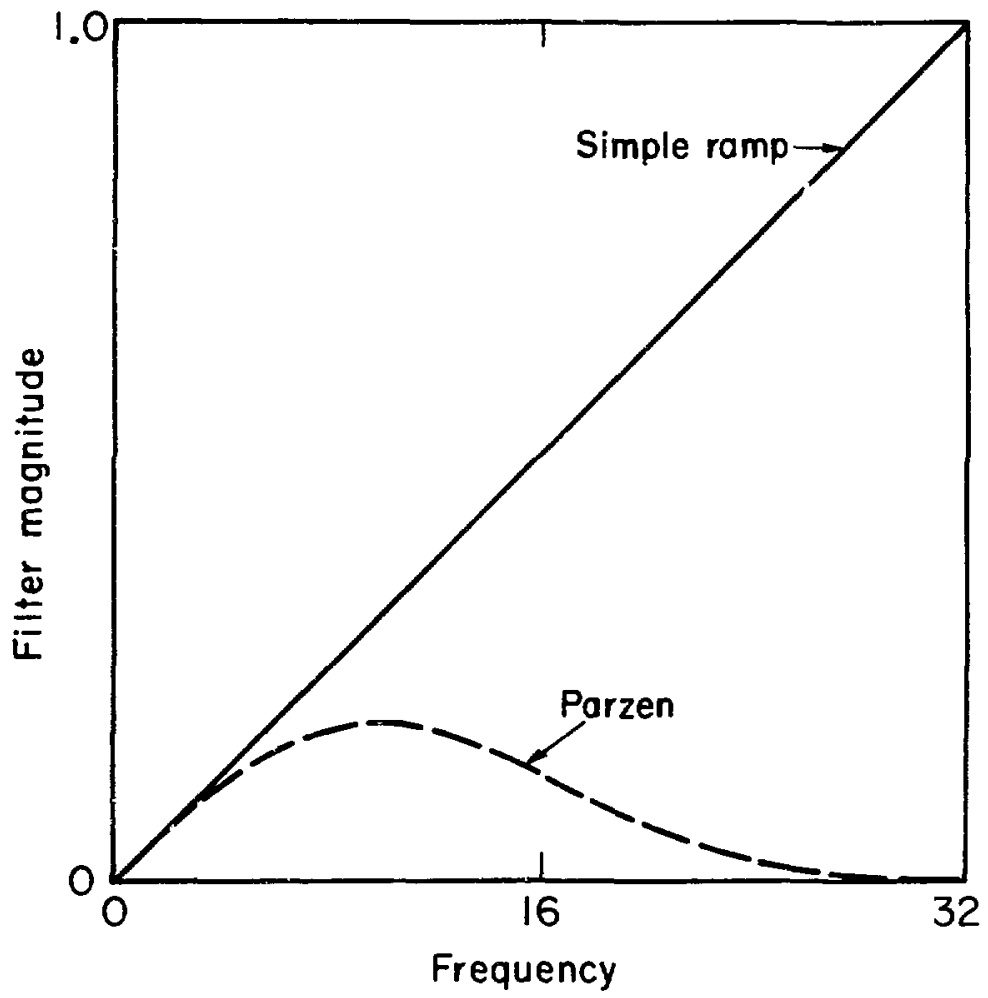

XBL7611-9463

Figure i0. Plot in frequency space of the Simple Ramp and Parzen filters. 
3. The adjoint transform of the point saurce projection function for both the parallel beam and fan beam geometry cannot be represented as a function with the form $h\left(x-x^{\prime}, y-y^{\prime}\right)$.

Points I. and 3. imply that only the back-projection operation gives an image which can be represented as a convolution and thus apolicable for the filter of the back-projection approach to transverse section reconstruction. A disadvantage of using this approach over other fan beam reconstruction methods which first filter and then back-project, is that a matrix which is four times the size of the reconstructed image is required so that the convolution result of one periad does not averlap the convolution result of the succeeding period when implementing the fast Fourier transfurm [19, Chapter 7]. This method may be faster; however, we have not yet compared it with other methocis.

The results using the filter of the back-projection approach to reconstructing fan beam data hints at a general approach to reconstructing projection data generated from nonparallel beams such as curved rays.

It is required that a back-projection operator exists which gives a point soure response which is either proportional to $1 /\left|r-\sim_{0}\right|$ or is a function which depends only on the radial distance from the point source. It is also conceivable that there are back-projection operators for three-dimensional jeometries such as the cone beam which give a back-projection image that can be deconvolved using the filter of the back-projection approach to reconstruction. 


\section{ACKNOWL EDGMENTS}

inis work was supported by the U.S. Energy Research and Development Administration. The basis of this paper was developed out of the work of Dr. Thomas Budinger. His encouragement and suggestions are greatly appreciated. I would also like to than! Dr. Ronald Huesman whose suggestions led to some of the key results presented. 


\section{BIBL IOGRAPHY}

1. T. F. Budinger and G. T. Gullberg, Paconstruction by Two-Dimensional Filtering of Simple Superposition Transverse-Section Image, in Imaging Processing for 2-D and 3-D Reconstruction from Projections, Stanford, August 4-7, 1975 (Opt. Soc. Am.) pp. ThA9-1 to 4.

2. R. H. T. Bates and T. M. Peters, Towards Improvements in Tomography, New Zealand J. Sci. 14 (1977), pp. 883-896.

3. T. M. Peters, Image Reconstruction from Projection, Ph.D. Thesis, University of Canterbury, New Zealand (1973).

4. P. R. Smith, T. M. Peters and R. H. T. Bates, Image Reconstruction from Finite Number of Projections, J. Phys. A. ㅁ (1973), pp. 361-382.

5. D. P. Boyd, Instrumentation Considerations for Fan Beam ComputerizedTomography of the Body, in Image Processing for 2-D and 3-D Reconstruction from Projections, Stanford, August 4-7, 1975 (Opt. Soc. Am.) pp. TUA6-1 to 4.

6. L. Wang and 2. H. Cho, 3-D Reconstruction Algorithms for Fan Beam Scans, in Image Processing for $2-D$ and $3-D$ Reconstruction from Projections, Stanford, August 4-7, 1975 (Opt. Soc. Am.) pp. WB6-1 to 4.

7. G. T. Herman, A. V. Lakshminarayanan and A. Naparstek, Convolution Reconstruction Techniques for Divergent Beams, Comput. Bio1. Med., 1976, in press.

8. S. Genna, S. C. Pang and B. A. Burrows, Application of Fan Reconstruction Geonetries to Transmission and Emission Systems, in Reconstructi se Tomography in Diagnostic Radiology and Nuclear Medicine, Ed. M. M. Ter-Pogossian (Baltimore, University Park), in press.

9. S. C. Pang and S. Genna, A Fourier Convolution Fan-Geometry Reconstruction Algorithm: Simulation Studies, Noise Propagation, and 
Polychromatic Degradation, to appear.

10. P. Stonestrom and A. Macovski, The Scatter Problem in Fan Beam Computerized Tomographic Systems, in Image Processing for 2-D and 3-D Reconstruction from Projections, Stanford, August 4-7, 1975 (Opt. Soc. Am.) pp. WB3-1 to 4 .

11. J. W. Beattie, Tomographic Reconstruction from Fan Beam Geometry Using Radon's Integration Method, IEEE Trans. Nuc?. Sci. NS-22 (1975) pp. 359-363.

12. T. M. Peters, Image Reconstruction from Projections Measured with Diverging Rays, in Techniques of Three-Dimensional Reconstruction, Ed. R. B. Marr (Proceedings of an Internationa? Workshop at Brookhaven National Laboratory, Upton, New York, July 16-19, 1974) pp. 152-153.

13. M. Ein-Ga?, The Shadow Transform: An Approach to Cross-Sectional Imaging, Information Systems Laboratory Technical Report 6351-1, Stanford University (November 1974).

14. R. B. Marr, On the Reconstruction of a Function on a Circular Domain from a Sampling of Its Line Integrals, J. Math. Anal, and Appl. $\underline{45}$ (1974) pp. 357-374.

15. P.F.C. Gilbert, The Reconstruction of a Three-Dimensional Structure from Projections and Its Application to Electron Microscopy II. Direct Methods, Proc. Roy. Soc. (London) Ser. B, 182 (1972) pp. 39-102.

16. T. F. Budinger and G. T. Gullberg, Three-Dimensional Reconstruction in Nuclear Medicine by Iterative Least-Squares and Fourier Transform Techniques, IEEE Trans. Nucl. Sci. NS-21 (1974) pp. 2-20.

17. R. R. Goldberg, Methods of Real Analysis, Blaisdell Publishing Co., New York, 1964. 
$-55-$

18. A. Papoulis, Systems and Transforms with Applications in Optics, McGraw-Hill, New York, 1968.

19. E. 0. Brigham, The Fast Fourier Transform, Prentice-Hall, Englewood Cliffs, New Jersey, 1974. 